\title{
Tissue Kallikrein Alleviates Cerebral Ischemia-Reperfusion Injury by Activating the B2R-ERK1/2-CREB-Bcl-2 Signaling Pathway in Diabetic Rats
}

\author{
Ruifeng Shi, ${ }^{1,2}$ Kunxiong Yuan, ${ }^{3}$ Bin Hu, ${ }^{4}$ Hongfei Sang, ${ }^{1}$ Lizhi Zhou, ${ }^{5}$ Yi Xie, ${ }^{1}$ \\ Lili Xu, ${ }_{1}^{1}$ Qinqin Cao, ${ }^{1}$ Xin Chen, ${ }^{1}$ Lingling Zhao, ${ }^{1}$ Yunyun Xiong, ${ }^{1}$ Gelin Xu, \\ Xinfeng Liu, ${ }^{1}$ Ling Liu, ${ }^{1}$ and Renliang Zhang ${ }^{1}$ \\ ${ }^{1}$ Department of Neurology, Jinling Hospital, Medical School of Nanjing University, Nanjing, Jiangsu 210002, China \\ ${ }^{2}$ Department of Neurology, Maanshan Municipal People's Hospital, Maanshan, Anhui 243000, China \\ ${ }^{3}$ Department of Neurology, Jinling Hospital, Southern Medical University, Nanjing, Jiangsu 210002, China \\ ${ }^{4}$ Department of Orthopedics, Maanshan Municipal People's Hospital, Maanshan, Anhui 243000, China \\ ${ }^{5}$ State Key Laboratory of Organ Failure Research, National Clinical Research Center for Kidney Disease, Department of \\ Biostatistics, School of Public Health and Tropical Medicine, Southern Medical University, 510515 Guangzhou, China
}

Correspondence should be addressed to Ling Liu; liuling8210@126.com and Renliang Zhang; zhangrenliang@nju.edu.cn

Received 23 February 2016; Accepted 6 June 2016

Academic Editor: Angel Catalá

Copyright (c) 2016 Ruifeng Shi et al. This is an open access article distributed under the Creative Commons Attribution License, which permits unrestricted use, distribution, and reproduction in any medium, provided the original work is properly cited.

\begin{abstract}
Diabetes mellitus (DM) substantially increases the risk of ischemic stroke and reduces the tolerance to ischemic insults. Tissue kallikrein (TK) has been demonstrated to protect neurons from ischemia/reperfusion (I/R) injury in orthoglycemic model by activating the bradykinin B2 receptor (B2R). Considering the differential effects of B2R or bradykinin B1 receptor (B1R) on cardioprotection and neuroprotection in $\mathrm{I} / \mathrm{R}$ with or without diabetes, this study was designed to investigate the role of TK during cerebral I/R injury in streptozotocin-induced diabetic rats. Intravenous injection of TK inhibited apoptosis in neurons, alleviated edema and inflammatory reactions after focal cerebral $I / R$, significantly reduced the infarct volume, and improved functional recovery. These beneficial effects were accompanied by activation of the extracellular signal-regulated kinase 1/2 (ERK1/2), cAMP response element-binding (CREB), and Bcl-2 signal proteins. Inhibition of the B2R or ERK1/2 pathway abated the effects of TK, whereas an antagonist of B1R enhanced the effects. These findings reveal that the neuroprotective effect of TK against cerebral I/R injury in streptozotocin-induced diabetic rats mainly involves the enhancement of B2R and ERK1/2-CREB-Bcl-2 signaling pathway activity.
\end{abstract}

\section{Introduction}

Ischemic stroke is the third leading cause of disabilityadjusted life years worldwide [1]. Diabetes mellitus (DM) is one definitive risk factor of stroke and has been diagnosed in more than $30 \%$ of ischemic stroke patients [2]. Indeed, stroke survivors with diabetes have a higher risk of recurrence and a poorer prognosis compared with nondiabetics [3-6]. Although intensive glycemic control decreases the infarct volume and improves functional recovery after stroke [7], early recanalization is the only evidence-based effective therapy for improving the clinical outcomes of acute ischemic stroke [8]. Ischemia/reperfusion (I/R) injury, as one serious complication of restoring blood flow to the ischemic cerebrum, may offset the benefits of recanalization, especially in diabetes cases [9]. DM is known to exacerbate ischemic injury and to impede functional recovery by elevating inflammatory cytokines, promoting leukocyte infiltration, and accelerating the subsequent apoptotic cascade and neuronal death $[5,10-$ 12]. Moreover, the cerebrum of diabetics is resistant to the neuroprotective effects of ischemic pre- or postconditioning or the administration of neuropharmacological agents [13]. Thus, further evaluation of the effects of DM is necessary to develop novel protective therapies for cerebral ischemia. 
Kinins derived from kininogen produced by tissue kallikrein (TK) exert a broad spectrum of cellular functions via activation of the bradykinin $\mathrm{B} 1$ or $\mathrm{B} 2$ receptor $(\mathrm{B} 1 \mathrm{R}$ or $\mathrm{B} 2 \mathrm{R}$, resp.) [14]. The physiological and pathological effects of TK have been shown to involve several cellular signaling pathways. Our previous studies have shown that TK could alleviate glutamate-induced neurotoxicity and protect cortical neurons against I/R and hypoxia/reoxygenation injury via the extracellular signal-regulated kinase $1 / 2($ ERK1/2) pathway [15-17]. In nondiabetic animals, TK was shown to improve neurofunctioning after ischemic stroke by inhibiting the NF- $\kappa \mathrm{B}$ signal pathway, activating the ERK1/2 pathway [18, 19], while in diabetic rats, TK attenuates insulin resistance and diabetic nephropathy via activation of phosphatidylinositol 3-kinase/protein kinase B [20]. Furthermore, increased TK plasma concentrations might exert greater cardioprotection in type $2 \mathrm{DM}$ patients than that in non-DM patients [21]. Recently, we found a detrimental role of B1R and a beneficial effect of B2R in diabetic cerebral ischemia [22]. The present study aimed to assess whether TK could ameliorate cerebral I/R injury in streptozotocin-induced diabetic rats and investigate the roles of the ERK1/2 signaling pathway during acute ischemia and early reperfusion.

\section{Materials and Methods}

2.1. Establishment of the Diabetes Model. Male SpragueDawley rats (100-110 g) were purchased from the Animal Center of Jinling Hospital. All procedures were performed in accordance with the National Institutes of Health Guide for the Care and Use of Laboratory Animals (NIH Publications Number 80-23, revised 1996) and under the approval of the Institutional Animal Care and Use Committee of Nanjing University. All animals were placed on a 12/12-hour light/dark schedule and housed in a temperature- $\left(22 \pm 2^{\circ} \mathrm{C}\right)$ and humidity-controlled $(55 \pm 5 \%)$ room with free access to food (high-fat diet) and water. Diabetes was induced with streptozotocin (Sigma-Aldrich, St. Louis, MO, USA) at a dose of $35 \mathrm{mg} / \mathrm{kg}$ after three weeks of high-fat diet feeding, as previously described [23]. Two weeks after the injection of streptozotocin, rats with fasting blood glucose concentrations $\geq 16.67 \mathrm{mmol} / \mathrm{L}$ (300 $\mathrm{mg} / \mathrm{dL})$ in three separate measures were considered diabetic.

2.2. Focal Cerebral Ischemia. Middle cerebral artery occlusion (MCAO) and reperfusion models in rats were established as previously described [24]. After $90 \mathrm{~min}$ of MCAO, the filament was withdrawn to restore cerebral blood flow (CBF) through the left MCA. CBF was monitored continuously with a PeriFlux Laser Doppler System 5000 (Perimed $\mathrm{AB}$, Sweden) throughout the operation to confirm proper occlusion and reperfusion. After full recovery from anesthesia, rats were tested with the Longa score. The scores of 1 (failure to extend left forepaw fully), 2 (circling to the left), and 3 (falling to the left) represent mild focal neurologic deficit, moderate focal neurologic deficit, and severe focal deficit, respectively. The animal with a score of 4 could not walk spontaneously and experienced coma and confusion.
Sham

\section{Without ischemia}

Saline

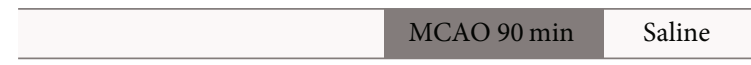

TK

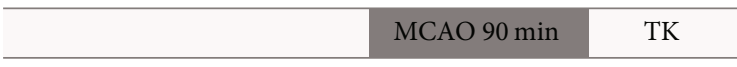

$\mathrm{B} 1 \mathrm{RA}+\mathrm{TK}$

\begin{tabular}{lll}
\hline BIR antagonist & $30 \mathrm{~min}$ & $\mathrm{MCAO} 90 \mathrm{~min}$ \\
\hline
\end{tabular}

$\mathrm{B} 2 \mathrm{RA}+\mathrm{TK}$

\begin{tabular}{l|l|l|l}
\hline B2R antagonist & $30 \mathrm{~min}$ & MCAO 90 min & TK \\
\hline
\end{tabular}

$\mathrm{B} 1 \mathrm{RA}+\mathrm{B} 2 \mathrm{RA}+\mathrm{TK}$

\begin{tabular}{|c|c|c|c|}
\hline $\begin{array}{l}\text { B1R antagonist } \\
B 2 R \text { antagonist }\end{array}$ & $30 \mathrm{~min}$ & MCAO $90 \mathrm{~min}$ & TK \\
\hline
\end{tabular}

DMSO

\begin{tabular}{|c|c|c|}
\hline DMSO & $5 \mathrm{~min}$ & MCAO $90 \mathrm{~min}$ \\
\hline
\end{tabular}

U0126

\begin{tabular}{l|l|l|l|}
\hline DMSO+U0126 & $5 \mathrm{~min}$ & MCAO $90 \mathrm{~min}$ & Saline \\
\hline
\end{tabular}

U0126+TK

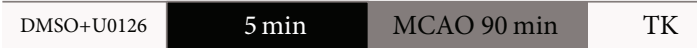

FIGURE 1: The experimental grouping and protocol.

The rats that attained a score of 0 were excluded from the study, which indicated noneurologic deficit. Sham-operated rats underwent the same operation without the insertion of the filament.

2.3. Experimental Groups. Animals were randomized into nine groups: sham operation, saline, TK, TK+B1RA (B1R antagonist), TK+B2RA (B2R antagonist), $\mathrm{TK}+\mathrm{B} 1 \mathrm{RA}+\mathrm{B} 2 \mathrm{RA}$, DMSO, U0126 (ERK1/2 inhibitor), and TK+U0126. Either the B2R antagonist bradyzide ( $1 \mathrm{nmol} / \mathrm{kg}$, Sigma-Aldrich) or B1R antagonist Lys-(des-Arg9-Leu8)-bradykinin (300 nmol/kg, Sigma-Aldrich) was injected $30 \mathrm{~min}$ before the operation, whereas TK $\left(1.6 \times 10^{-2}\right.$ PNAU/kg, Techpool Bio-Pharma Co. Ltd., Canton, China) [25] or saline $(2 \mathrm{~mL} / \mathrm{kg})$ was given intravenously immediately after reperfusion. U0126 (9903, Cell Signaling Technology, Danvers, USA), an inhibitor of the ERK1/2 signaling pathway, was dissolved in dimethyl sulfoxide (DMSO, Sigma-Aldrich, St. Louis, MO, USA) at $10 \mathrm{mmol} / \mathrm{L}$ and was subsequently diluted with saline for use. U0126 $(400 \mu \mathrm{g} / \mathrm{kg})$ or an equal amount of DMSO was administered intravenously $5 \mathrm{~min}$ before the operation [26]. The experimental grouping and protocol are described in the flow diagram (Figure 1). Previous studies demonstrated that the expression of $\mathrm{B} 1 \mathrm{R}$ and $\mathrm{B} 2 \mathrm{R}$ in cerebral ischemia/reperfusion injury peaks at $24 \mathrm{~h}$ after reperfusion in diabetes [22]. Therefore, $24 \mathrm{~h}$ after reperfusion was chosen as the time point for research in this study. 
2.4. Assessment of Neurological Deficits. Neurological deficits were evaluated by an investigator blinded to the groupings at $24 \mathrm{~h}$ after reperfusion using the neurological severity scores (NSS) [27], which included motor test, sensory test, beam balance test, and examinations of reflexes and abnormal movements.

2.5. Estimation of Brain Edema. Rats were sacrificed $24 \mathrm{~h}$ after reperfusion. The hemispheres were weighed to obtain the wet weight (WW), and their dry weights (DW) were measured after desiccation at $105^{\circ} \mathrm{C}$ for $24 \mathrm{~h}$. The brain moisture content (\%) was calculated as previously described [22].

2.6. Evaluation of Infarct Volume. Brains were removed $24 \mathrm{~h}$ after reperfusion and cut into six $2 \mathrm{~mm}$ thick coronal slices. The infarct volume was evaluated by 2,3,5-triphenyltetrazolium chloride (TTC, Sigma-Aldrich, St. Louis, MO, USA) staining and analyzed according to our previous protocol [22].

2.7. Histopathological Study. For histopathological study, rats were perfused with $200 \mathrm{~mL}$ of $0.9 \%$ saline and $200 \mathrm{~mL}$ $4 \%$ paraformaldehyde (PFA, pH 7.4) successively through the left ventricle $24 \mathrm{~h}$ after reperfusion. Then, the cerebral hemisphere was collected and fixed with 4\% PFA. After gradient elution with sucrose, the brain was quickly frozen and cut into $14 \mu \mathrm{m}$ sections.

2.7.1. Fluoro-Jade C Staining (FJC). Slides were immersed in a basic alcohol solution consisting of $1 \% \mathrm{NaOH}$ in $80 \%$ ethanol for $5 \mathrm{~min}$ and were then rinsed in $70 \%$ ethanol for $2 \mathrm{~min}$, followed by incubation in $0.06 \%$ potassium permanganate solution for $10 \mathrm{~min}$. The sections were then immersed in $0.0001 \%$ working solution of FJC (dissolved in $0.1 \%$ acetic acid vehicle) for $15 \mathrm{~min}$. After washing and drying, the sections were cleaned in xylene and cover-slipped with DPX (Sigma-Aldrich, St. Louis, MO, USA) nonfluorescent mounting media. The data are expressed as the number of FJC-positive cells counted per section relative to the sham group.

2.7.2. TUNEL Staining. The terminal deoxynucleotidyl transferase-mediated dUTP nick-end labeling (TUNEL) assay (Roche, Indianapolis, IN, USA) was used to assess apoptosis in neurons. Slides were postfixed with 4\% PFA for $20 \mathrm{~min}$ and permeabilized with $0.1 \%$ Triton X-100 for 2 min on ice. After incubating in $15 \%$ glacial acetic acid for $2 \mathrm{~min}$, the sections were treated with TUNEL mixture (enzyme solution : label solution $=1: 9$ ) for $60 \mathrm{~min}$ at $37^{\circ} \mathrm{C}$ in a dark humidified atmosphere, followed by converter-AP for $30 \mathrm{~min}$. After washing with PBS three times for $5 \mathrm{~min}$ each, the specimens were incubated with BICP/NBT (Alkaline Phosphatase Color Development Kit, Beyotime, Shanghai, China) for $30 \mathrm{~min}$ and mounted under glass coverslips with CC/MOUNT. The data were expressed and analyzed in the same manner as for FJC staining.
2.7.3. Immunohistochemical Examination. Slides were postfixed in $4 \%$ PFA for $15 \mathrm{~min}$ followed by $0.3 \% \mathrm{H}_{2} \mathrm{O}_{2}$ for $30 \mathrm{~min}$. After permeabilization with $0.1 \%$ Triton for $10 \mathrm{~min}$, the sections were incubated with $5 \%$ albumin bovine (Generay biotech, Shanghai, China) for $1 \mathrm{~h}$. Then, the sections were incubated overnight with the following primary antibodies: anti-ionized calcium-binding adapter molecule 1 (Ibal) (1:1,000, Wako, 019-19741, Osaka, Japan), anti-cleaved caspase-3 (1:100, Cell Signaling Technology, Danvers, USA), or anti-myeloperoxidase (MPO) $(1: 200$, Abcam, 65871 Cambridge, England). The slides were washed and then incubated with biotinylated anti-rabbit IgG antibody (Jackson, 711065152, West Grove, PA, USA) $(1: 200)$ for $2 \mathrm{~h}$ at room temperature. After incubation with $\mathrm{ABC}$ solution (Vector labs, California, USA), the slides were stained with the DAB Peroxidase Substrate Kit (Vector labs, California, USA), followed by PBS washing once the desired color was achieved. Sections were cleaned in xylene and cover-slipped with neutral balsam. The data were expressed and analyzed in the same manner as for FJC staining.

2.8. Real-Time Quantitative Reverse Transcription Polymerase Chain Reaction. Total RNA was extracted from ischemic boundary region tissue at $24 \mathrm{~h}$ after reperfusion using the Trizol reagent (Sigma-Aldrich, St. Louis, MO, USA) and then reverse-transcribed into cDNA using a RevertAid First Strand cDNA Synthesis kit (Thermo Scientific, Waltham, MA, USA) following the manufacturer's recommendations. Quantitative real-time PCR was performed using a realtime PCR system (Agilent Technologies, Santa Clara, CA, USA) with a fluorescent dye (CW0956 UltraSYBR Mixture). Glyceraldehyde-3-phosphate dehydrogenase (GAPDH) was used as an endogenous reference gene. The normalized messenger RNA levels were previously described in detail [28]. Data are expressed as the ratio of the level of IL- $1 \beta$ or TNF- $\alpha$ to that of GAPDH in the treated groups relative to the sham group. The primer sequences for the genes were as follows: IL-1 $\beta$ : sense: $5^{\prime}$-AGACTTCACAGAGGATACCACCCAC- $3^{\prime}$, antisense: $5^{\prime}$-CAATCAGAATTGCCATTGCACAA- $3^{\prime}$; TNF- $\alpha$ : sense: $5^{\prime}$-AGCAAACCACCAAGCGGAGG$3^{\prime}$, antisense: $5^{\prime}$-CAGCCTTGTCCCTTGAAGAGAAC- $3^{\prime}$; and GAPDH: sense: $5^{\prime}$-GCAAGTTCAACGGCACAG-3', antisense: $5^{\prime}$-GCCAGTAGACTCCACGACAT-3'.

2.9. Western Blotting. Samples for western blotting were collected from brain infarction boundary regions. After extraction and quantification, equal amounts of protein were separated by sodium dodecyl sulfate-PAGE and then transferred to an immobilon-polyvinylidene fluoride membrane. The membranes were blocked using 5\% milk for $2 \mathrm{~h}$ at room temperature and probed with the primary antibody overnight at $4^{\circ} \mathrm{C}$. These primary antibodies included rabbit anti-phospho-ERK1/2 (1:5,000), rabbit anti-total-ERK1/2 $(1: 5,000)$, rabbit anti-phospho-cAMP response elementbinding protein $(\mathrm{CREB})(1: 1,000)$, rabbit anti-total-CREB $(1: 1,000)$, rabbit anti-Bcl-2 $(1: 200)$, rabbit anti-Bax $(1: 400)$, rabbit anti-cleaved caspase- $3(1: 1000)$, and rabbit anti- $\beta$ actin $(1: 5,000)$. The secondary antibody was anti-rabbit 
HRP-conjugated antibody $(1: 4,000)$. All primary antibodies were purchased from Cell Signaling Technology (Danvers, USA), with the exception of the $\mathrm{Bcl}-2$ antibody purchased from Santa Cruz (Dallas, USA). Immunoblots were developed with the Immobilon ECL method (Millipore, Billerica, Massachusetts, USA). Gray bands were converted to density values using Image J software for quantification analysis.

2.10. Data Analysis and Statistics. All data except for the NSS values are expressed as mean $\pm \mathrm{SD}$. The neurologic tests scores were analyzed using the Kruskal-Wallis test followed by the Mann-Whitney $U$ test with Bonferroni corrections. Differences between groups were compared using one-way analysis of variance with the Welch correction for unequal variances. Post hoc tests, such as Bonferroni or Dunnett's T3 multiple comparison tests, were used according to whether unequal variances were present. A two-sided $P$ value of $P<$ 0.05 was defined as significant.

\section{Results}

3.1. Modifications of Neurological Deficits. At $24 \mathrm{~h}$ after $\mathrm{MCAO}$, neurologic function improved in the TK group compared with the saline group $(P<0.05$, Figure $2(\mathrm{a}))$. Compared with the TK group, the B1RA+B2RA+TK and $\mathrm{B} 2 \mathrm{RA}+\mathrm{TK}$ groups showed higher neurological deficit scores $(P<0.05)$, whereas the B1RA+TK group showed a comparable score $(P>0.05)$. Furthermore, neurological function in the B1RA+B2RA+TK group was worse than that of the B1RA+TK group $(P<0.05)$ but similar to that of the B2RA+TK group $(P>0.05)$. Treatment with the ERK1/2 inhibitor U0126 significantly aggravated neurological functioning $(P<0.05)$ and compromised the beneficial effect of TK (TK versus U0126+TK, $P<0.05$ ).

3.2. Variation of Brain Edema. Brain edema at $24 \mathrm{~h}$ after reperfusion was significantly alleviated in TK group compared to saline group $(P<0.05$, Figure $2(\mathrm{~b}))$. B1R antagonist pretreatment had no interference on TK. However, pretreatment with the B2R antagonist hindered the efficacy of TK $(P<0.05)$. Brain edema in the B1RA+B2RA+TK group was more severe than that in the TK and B1RA+TK groups but less than that in the $\mathrm{B} 2 \mathrm{RA}+\mathrm{TK}$ group $(P<0.05)$. Administration of U0126 alone clearly increased the brain water content compared to rats in the saline group $(P<0.05)$. However, the brain water content in the U0126+TK group was not different from that in the TK group.

3.3. Change in Cerebral Infarct Volume. TK treatment remarkably reduced the infarct volume compared to saline at $24 \mathrm{~h}$ after reperfusion $(P<0.05$, Figures $2(\mathrm{c})$ and 2(d)). Administration of B1RA+TK could further reduce the infarct volume of TK-treated animals, whereas B2RA+TK or B1RA+B2RA+TK treatment abolished the beneficial effect observed with the TK intervention $(P<0.05)$. Additionally, the infarct volume in the B1RA+B2RA+TK group was lower than that in the B2RA+TK group but higher than that in the B1RA+TK group $(P<0.05)$. Treatment with the ERK1/2 inhibitor U0126 increased the infarct volume in the saline group $(P<0.05)$. Furthermore, the infarct volume with U0126+TK treatment was higher than that with TK treatment alone $(P<0.05)$.

3.4. Expression of Ibal, MPO, and Proinflammatory Cytokines. Ibal is a protein biomarker expressed in microglia that is upregulated during inflammatory responses after stroke in the infarction border zone. MCAO followed by $24 \mathrm{~h}$ of reperfusion induced a $20.46 \pm 2.40$-fold increase in Ibalpositive cells. Compared to saline, TK intervention significantly suppressed the activation of Ibal $(P<0.05$, Figures $3(\mathrm{~A})$ and 3(B)). Furthermore, the efficacy of TK was enhanced by pretreatment with the B1R antagonist but was abolished by the B2R antagonist $(P<0.05)$. The number of Ibal-positive cells in the B1RA+B2RA+TK group was higher than that in the TK and B1RA+TK groups but lower than that in the B2RA+TK group $(P<0.05)$. In addition, U0126 treatment led to an increase in microglial activation (U0126 versus saline, $P<0.05$ ), while pretreatment with U0126 abated the effect of TK (U0126+TK versus TK, $P<0.05$ ). Neutrophil infiltration into the ischemic regions was investigated by immunohistochemical staining for MPO (Figures $3(\mathrm{C})$ and $3(\mathrm{D}))$, and the change in MPO expression was similar to that observed for Ibal.

Proinflammatory cytokines are expressed in the ischemic core in the early stage of the brain ischemic model. Reperfusion for $24 \mathrm{~h}$ after MCAO (saline group) led to a 61.27 $\pm 7.46-$ fold increase in IL- $1 \beta$ and a 30.71 \pm 9.64 -fold increase in TNF$\alpha$ compared with the sham group (Figures 4(a) and 4(b)). Treatment with TK ameliorated this upregulation compared with the saline group $(P<0.05)$. Compared to TK-treated animals, B1R antagonism prior to MCAO attenuated IL-1 $\beta$ expression, whereas B2R antagonism elevated the levels of IL-1 $\beta$ and TNF- $\alpha(P<0.05)$. Simultaneous antagonism of B1R and B2R before TK treatment induced much higher levels of IL-1 $\beta$ and TNF- $\alpha$ than TK intervention alone $(P<$ $0.05)$. Furthermore, the relative expression of IL- $1 \beta$ and TNF- $\alpha$ in the B1RA+TK group was lower than that in the $\mathrm{B} 1 \mathrm{RA}+\mathrm{B} 2 \mathrm{RA}+\mathrm{TK}$ group $(P<0.05)$, whereas the level of IL$1 \beta$ in the B1RA+B2RA+TK group was lower than that in the B2RA+TK group $(P<0.05)$. After injection of the ERK1/2 inhibitor U0126, the relative expression of TNF- $\alpha$ and IL$1 \beta$ was dramatically increased compared to the saline group $(P<0.05)$. Additionally, pretreatment with U0126 elevated the level of TNF- $\alpha$ in rats that received TK treatment (TK versus U0126+TK, $P<0.05)$.

3.5. Neuronal Degeneration and Apoptosis. Neuronal degeneration was evaluated by FJC staining. Relative to the sham operation group, $24 \mathrm{~h}$ of reperfusion after MCAO stimulated a $19.12 \pm 2.92$-fold increase in FJC-positive cells in the ischemic cortex. Administration of TK decreased the relative density of FJC-positive cells compared to saline $(P<0.05$, Figures 5(A) and 5(B)). The efficacy of TK treatment was enhanced with the B1R antagonist but reversed with the B2R antagonist $(P<0.05)$. The number of degenerated neurons in the B1RA+B2RA+TK group was significantly greater than 


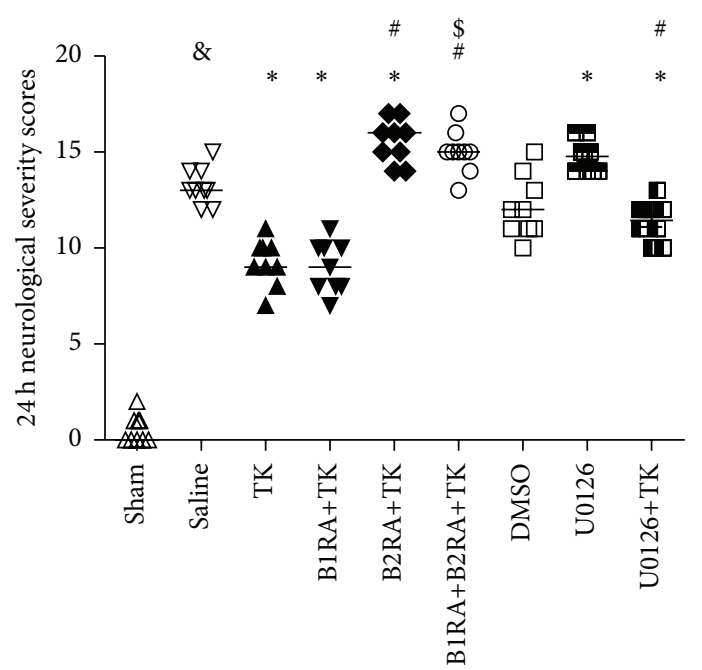

(a)

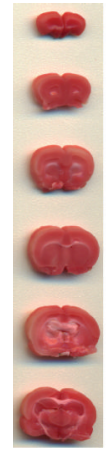

㞼
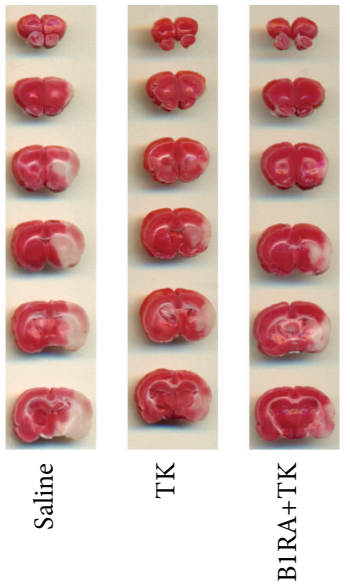

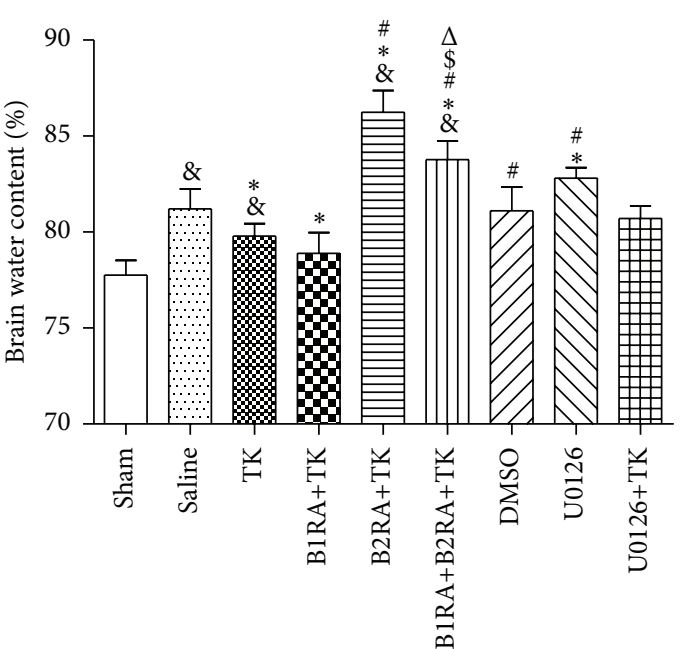

(b)
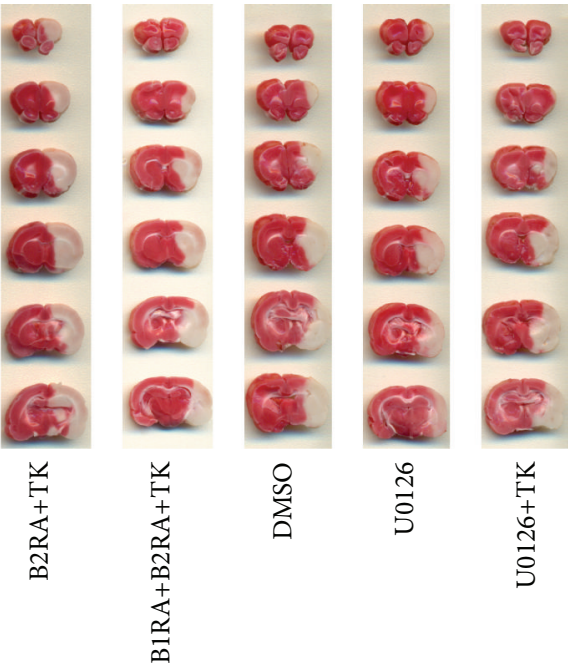

(c)

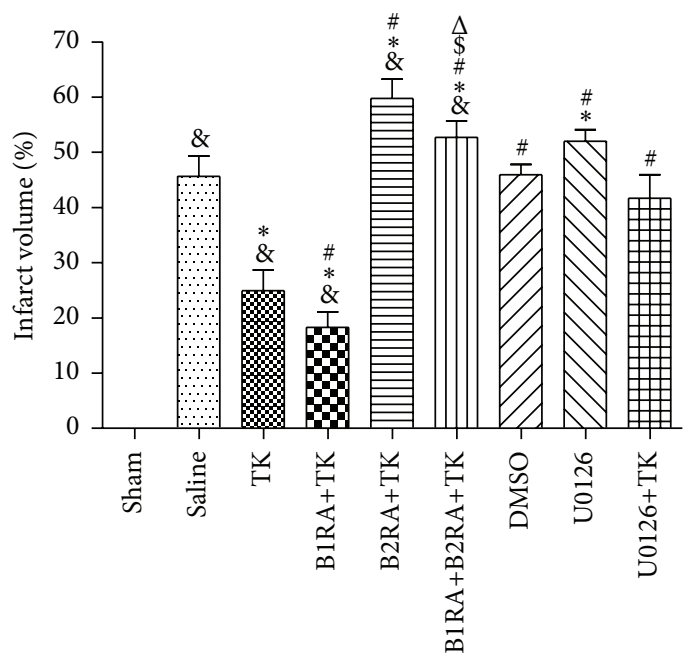

(d)

FIGURE 2: (a) NSS values recorded for each animal at $24 \mathrm{~h}$ after MCAO. $n=9$ rats/group. (b) Brain edema recorded in each animal at $24 \mathrm{~h}$ after MCAO. Data are expressed as mean $\pm \mathrm{SD} ; n=9$ rats/group. (c) 2,3,5-Triphenyltetrazolium chloride staining recorded in nine groups at $24 \mathrm{~h}$ after MCAO; (d) the infarct volume percent at $24 \mathrm{~h}$ after MCAO. Data are expressed as mean $\pm \mathrm{SD} ; n=7$ rats/group $\left({ }^{\&} P<0.05\right.$ versus sham group; ${ }^{*} P<0.05$ versus saline group; ${ }^{\#} P<0.05$ versus TK group; ${ }^{\$} P<0.05$ versus B1RA+TK group; ${ }^{\Delta} P<0.05$ versus B2RA+TK group). 

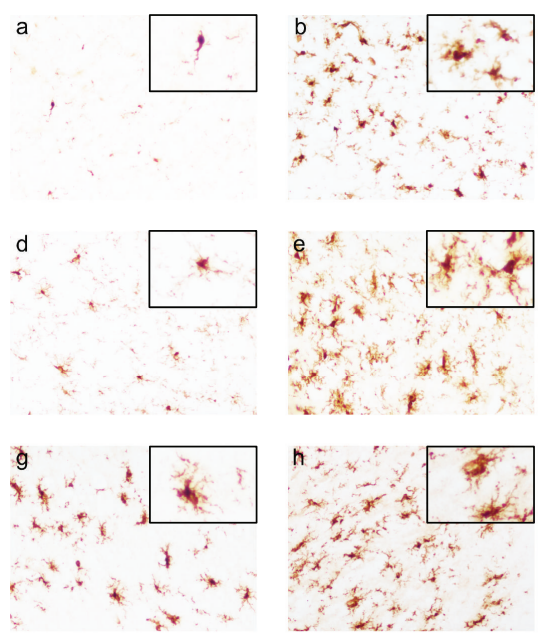

(A)
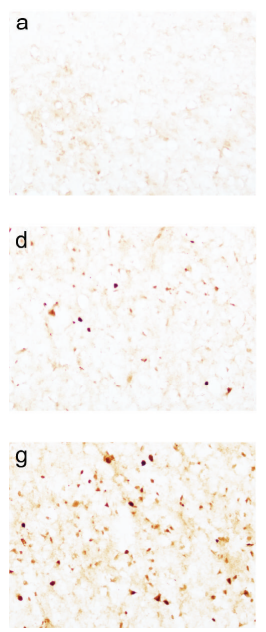
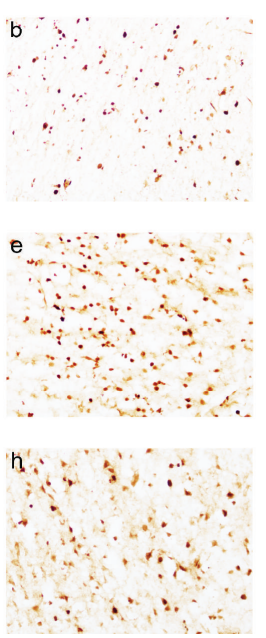

(C)
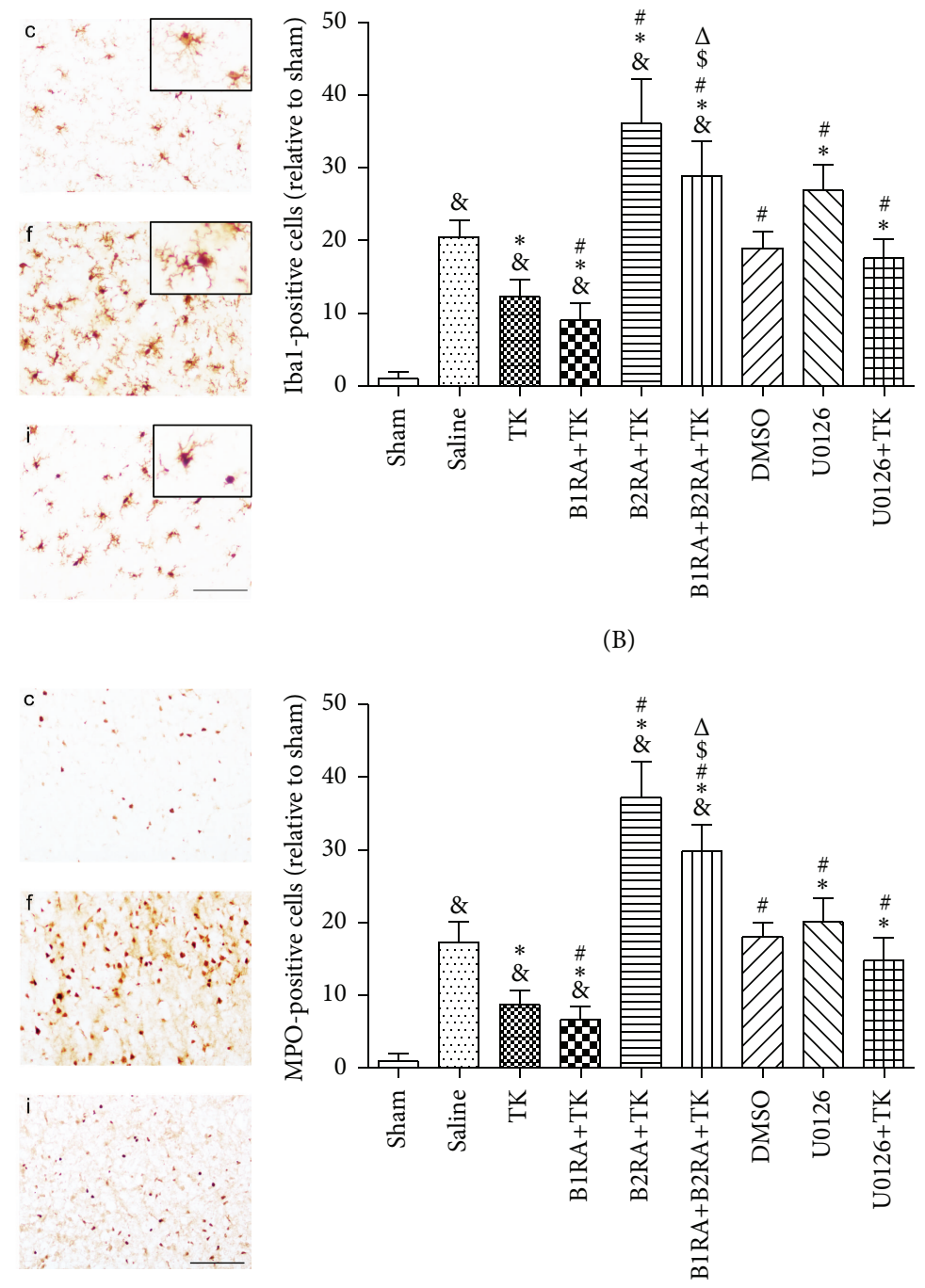

(D)

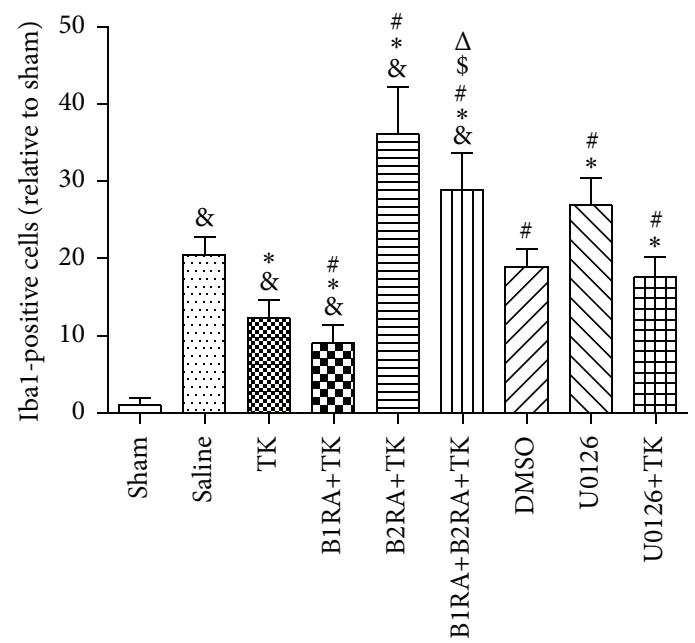

(B)

Figure 3: (A) Ibal staining was performed $24 \mathrm{~h}$ after MCAO to observe microglia. (B) The number of Ibal-positive cells was counted at $24 \mathrm{~h}$ after MCAO. (C) MPO staining was performed $24 \mathrm{~h}$ after MCAO to observe neutrophils. (D) The number of MPO-positive cells was counted $24 \mathrm{~h}$ after MCAO. (a) Sham; (b) saline; (c) TK; (d) B1RA+TK; (e) B2RA+TK; (f) B1RA+B2RA+TK; (g) DMSO; (h) U0126; (i) U0126+TK. Scale bar $=100 \mathrm{~mm}$. Data are expressed as mean $\pm \mathrm{SD} ; n=3$ rats/group. Six representative microscopic fields were analyzed for each rat $\left({ }^{\circledR} P<0.05\right.$ versus sham group; ${ }^{*} P<0.05$ versus saline group; ${ }^{\#} P<0.05$ versus TK group; ${ }^{\$} P<0.05$ versus B1RA+TK group; ${ }^{\Delta} P<0.05$ versus B2RA+TK group).

that in the TK and B1RA+TK groups but less than that in the B2RA+TK group $(P<0.05)$. U0126 pretreatment aggravated neuronal injury $(P<0.05)$ and weakened the benefit of TK $(P<0.05)$.

TUNEL staining and immunohistochemical staining for cleaved caspase- 3 were used to verify the antiapoptosis effect of TK in the ischemic cortex. The presence of apoptotic cells in each group was consistent with the extent of degenerated neurons (Figures 5(C), 5(D), 5(E), and 5(F)).

3.6. Activity of the ERK1/2 Signaling Pathway. Compared to corresponding expressions before MCAO, the relative levels of $\mathrm{p} / \mathrm{T}-\mathrm{ERK} 1 / 2$ and $\mathrm{p} / \mathrm{T}-\mathrm{CREB}$ showed remarkable increases with reperfusion; these levels showed fold increases of 2.6 and
2.8 after 1 h of reperfusion, respectively $(P<0.05$, Figure 6$)$. Thus, $1 \mathrm{~h}$ of reperfusion was chosen as the optimal time point for measuring the expression of signaling proteins.

TK treatment enhanced the relative expression of $\mathrm{p} / \mathrm{T}$ ERK1/2, p/T-CREB, and Bcl-2/ $\beta$-actin compared to the sham group at $1 \mathrm{~h}$ of reperfusion after MCAO, with higher values compared to the saline group $(P<0.05$, Figure 7$)$. Pretreatment with a B1R antagonist further elevated the levels of $\mathrm{p} / \mathrm{T}$-CREB and $\mathrm{Bcl}-2 / \beta$-actin in TK-treated animals $(P<$ $0.05)$. However, the $\mathrm{B} 2 \mathrm{R}$ antagonist abated the upregulation of $\mathrm{p} / \mathrm{T}$-ERK1/2, $\mathrm{p} / \mathrm{T}$-CREB, and $\mathrm{Bcl}-2 / \beta$-actin generated by TK treatment, and simultaneous pretreatment with the B1R and B2R antagonists had a similar effect $(P<0.05)$. However, the levels of all of the above proteins in the B1RA+TK group were higher than those in the B1RA+B2RA+TK group, whereas the 


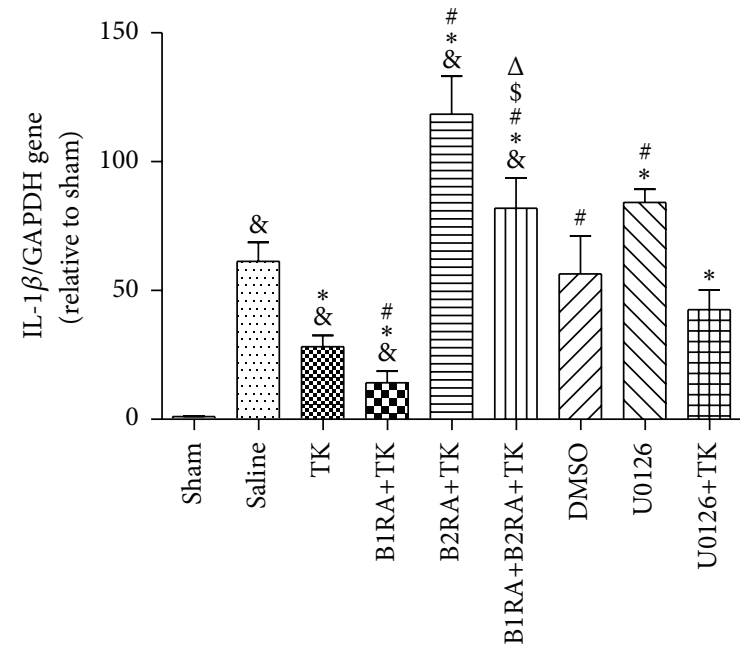

(a)

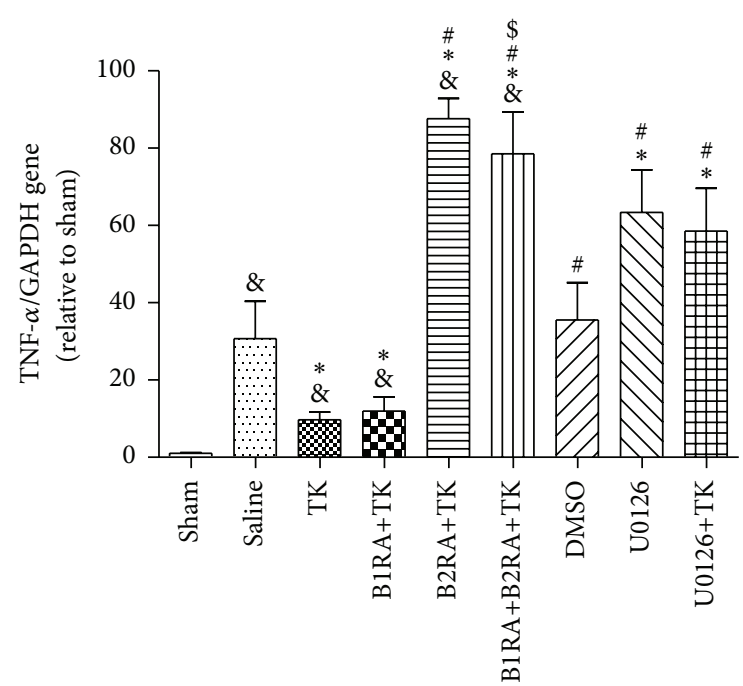

(b)

Figure 4: (a) The levels of IL-1 $\beta 24 \mathrm{~h}$ after MCAO. (b) The levels of TNF- $\alpha 24 \mathrm{~h}$ after MCAO. Values are expressed as mean \pm SD; $n=6$ rats/group $\left({ }^{\&} P<0.05\right.$ versus sham group; ${ }^{*} P<0.05$ versus saline group; ${ }^{\#} P<0.05$ versus TK group; ${ }^{\$} P<0.05$ versus B1RA+TK group; ${ }^{\Delta} P<0.05$ versus B2RA+TK group).

$\mathrm{p} / \mathrm{T}$-ERK1/2 and Bcl-2/ $\beta$-actin levels in the B2RA+TK group were significantly lower $(P<0.05)$. In contrast, the elevated levels of Bax and cleaved caspase- 3 expression stimulated by I/R were downregulated by TK treatment $(P<0.05)$. The inhibitory effect of TK on Bax expression was further amplified by the B1R antagonist but was weakened by the B2R antagonist $(P<0.05)$. The suppression effect of TK on cleaved caspase-3 was not changed significantly by B1R antagonist but impaired by B2R antagonist $(P<0.05)$. Furthermore, administration of B1RA $+\mathrm{B} 2 \mathrm{RA}+\mathrm{TK}$ decreased the levels of Bax and cleaved caspase- 3 compared to those observed in the B2RA+TK group but elevated the levels in comparison to the B1RA+TK group $(P<0.05)$.

Compared to saline treatment, injection of U0126 before MCAO resulted in decreased expression of $\mathrm{p} / \mathrm{T}-\mathrm{ERK} 1 / 2, \mathrm{p} / \mathrm{T}-$ CREB, and $\mathrm{Bcl}-2 / \beta$-actin compared to the saline group $(P<$ 0.05 , Figure 7). Additionally, U0126 treatment increased the levels of Bax and cleaved caspase- 3 relative to the saline group $(P<0.05)$. Moreover, pretreatment with U0126 impaired the effects of TK treatment $(P<0.05)$. During the experiments, the total immunoreactive ERK1/2 and CREB levels were rarely changed by MCAO surgery or other interventions.

\section{Discussion}

In this study, we observed the neuroprotective effects of TK on I/R injury in streptozotocin-induced diabetic rats, involving the reduction of brain edema and infarct volume, suppression of inflammation and apoptosis, and amelioration of ischemia-induced behavioral deficits. TK led to upregulated expression of $\mathrm{p}$-ERK1/2, $\mathrm{p}$-CREB, and $\mathrm{Bcl}-2$ proteins and downregulated expression of Bax and cleaved caspase3 proteins in the cerebral I/R injury of diabetic rats. Additionally, the neuroprotective effects of TK were promoted by a B1R antagonist but were abrogated by treatment with a B2R antagonist or ERK1/2 inhibitor.

Early inflammation contributes to brain damage following I/R. Activated microglia and neutrophils are central to the inflammatory response and can release cytokines. Furthermore, neuronal degeneration and apoptosis and inflammatory responses in ischemic areas can be aggravated by hyperglycemia [29]. Previous studies have shown that hyperglycemia enhances microglia activation and neutrophil migration, which further worsened postischemia injury $[30,31]$. This study revealed that immediate TK treatment after MCAO attenuated I/R-induced neuronal degeneration and cellular apoptosis, inhibited neutrophil migration and microglia activation, and reduced the levels of proinflammatory cytokines in diabetic rats. Furthermore, there were notably improved neurological outcomes in the TK-treated rats, indicating TK's involvement in the brain I/R process.

Pharmacological B2R blockade before TK treatment could boost neuron loss, microglia activation, neutrophil migration, and the levels of proinflammatory cytokines, suggesting that the neuroprotective effects of TK were mainly mediated by B2R. Our observations are consistent with the published literature showing that TK protects neurons from ischemic injury through activation of B2R [32]. Additionally, TK was demonstrated to inhibit I/R-induced apoptosis and inflammation in the brains of nondiabetic rats, and the efficacy was blocked by B2R antagonists [33]. However, in the present study, blockade of B1R before MCAO facilitated the actions of $\mathrm{TK}$, indicating that activation of $\mathrm{B} 1 \mathrm{R}$ exaggerated cerebral I/R injury in diabetic rats. Our recent findings showed that the upregulation of B1R in diabetic rats aggravated cerebral I/R injury compared with nondiabetic rats. Therefore, the B1R pharmacological antagonist plays a neuroprotective role in acute ischemia in diabetic rats [22], 

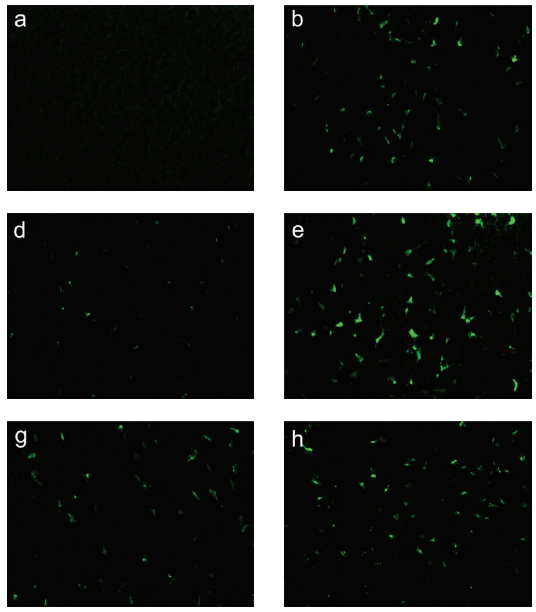

(A)
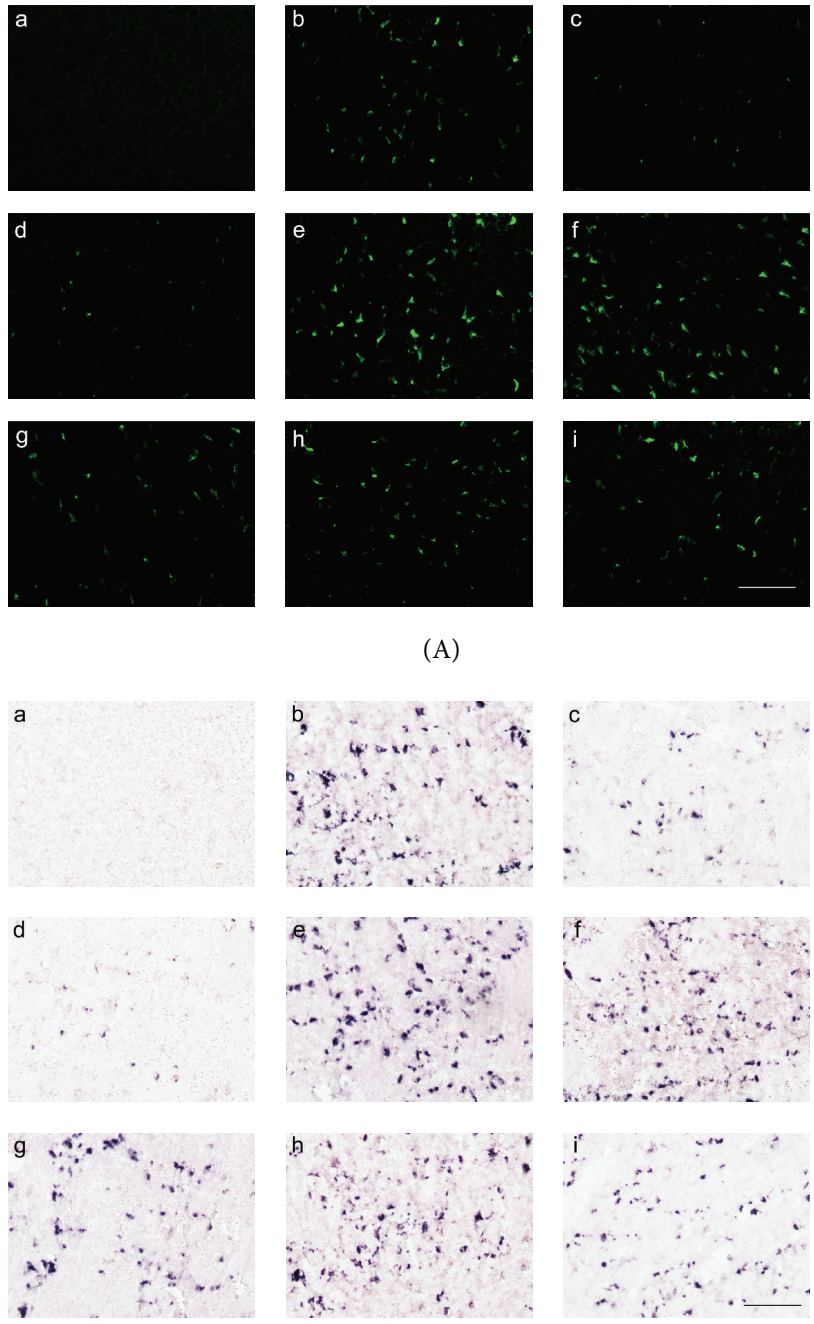

(C)
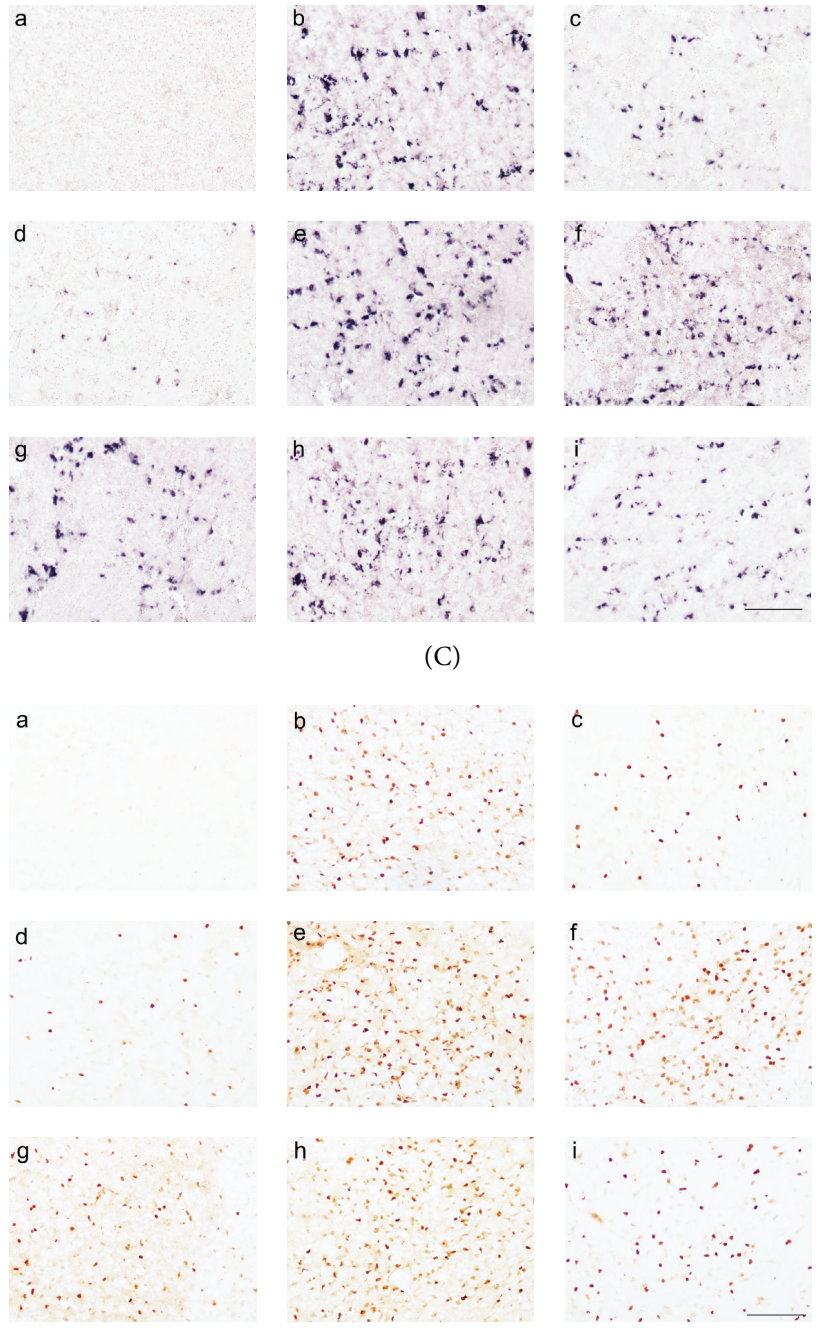

(E)

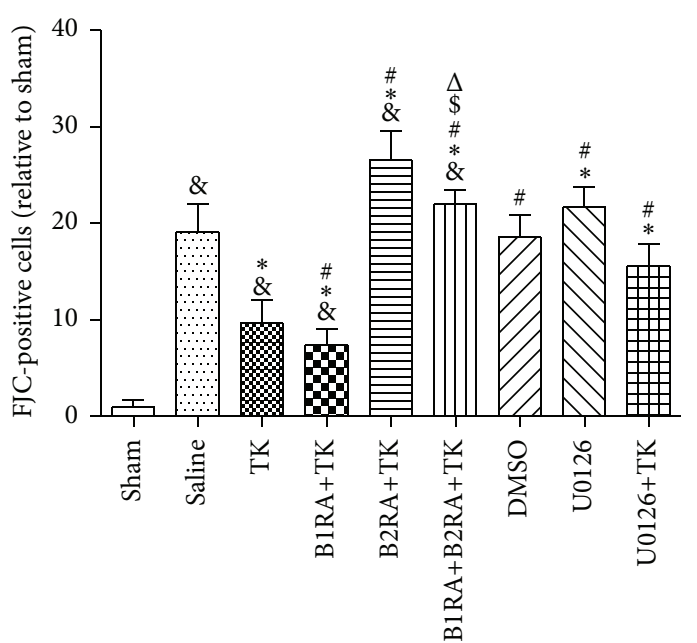

(B)

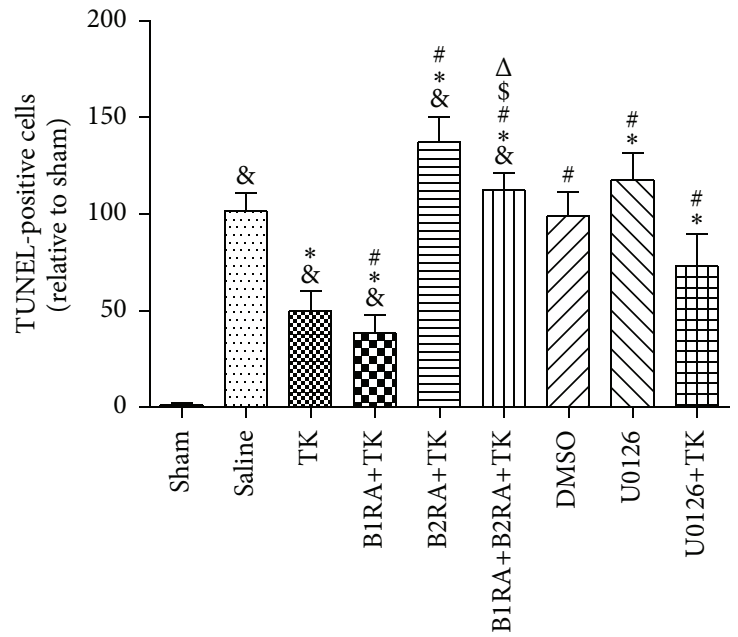

(D)

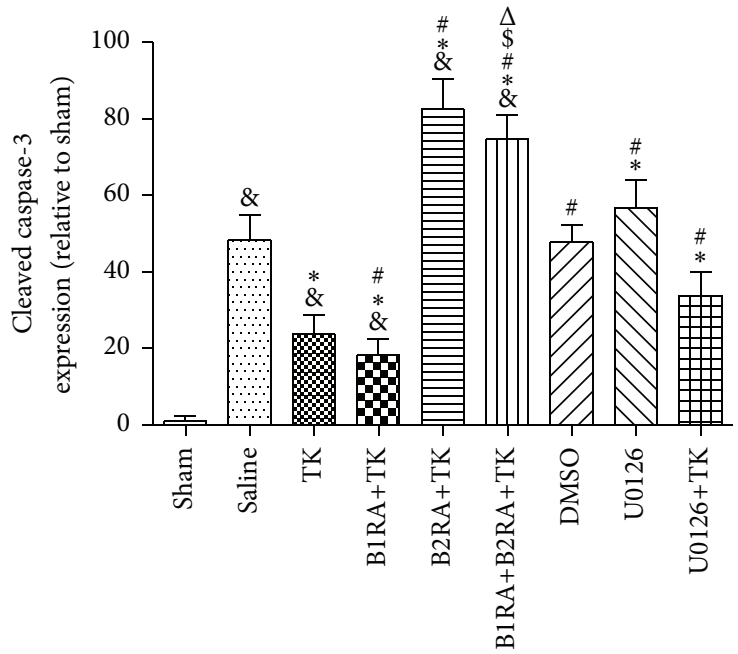

(F)

Figure 5: (A) FJC staining was performed $24 \mathrm{~h}$ after MCAO to assess neuron degeneration. (B) The number of FJC-positive cells was counted $24 \mathrm{~h}$ after MCAO. (C) TUNEL staining was performed $24 \mathrm{~h}$ after MCAO to assess apoptosis in neurons. (D) The number of TUNELpositive cells was counted $24 \mathrm{~h}$ after MCAO. (E) Cleaved caspase-3 staining was performed $24 \mathrm{~h}$ after MCAO to assess neuron apoptosis. (F) The number of cleaved caspase-3-positive cells was counted after MCAO. (a) Sham; (b) saline; (c) TK; (d) B1RA+TK; (e) B2RA+TK; (f) B1RA+B2RA+TK; (g) DMSO; (h) U0126; (i) U0126+TK. Scale bar $=100 \mathrm{~mm}$. Data are expressed as mean \pm SD; $n=3$ rats/group. Six representative microscopic fields were analyzed for each rat $\left({ }^{\&} P<0.05\right.$ versus sham group; ${ }^{*} P<0.05$ versus saline group; ${ }^{\sharp} P<0.05$ versus TK group; ${ }^{\$} P<0.05$ versus B1RA+TK group; ${ }^{\Delta} P<0.05$ versus B2RA+TK group). 


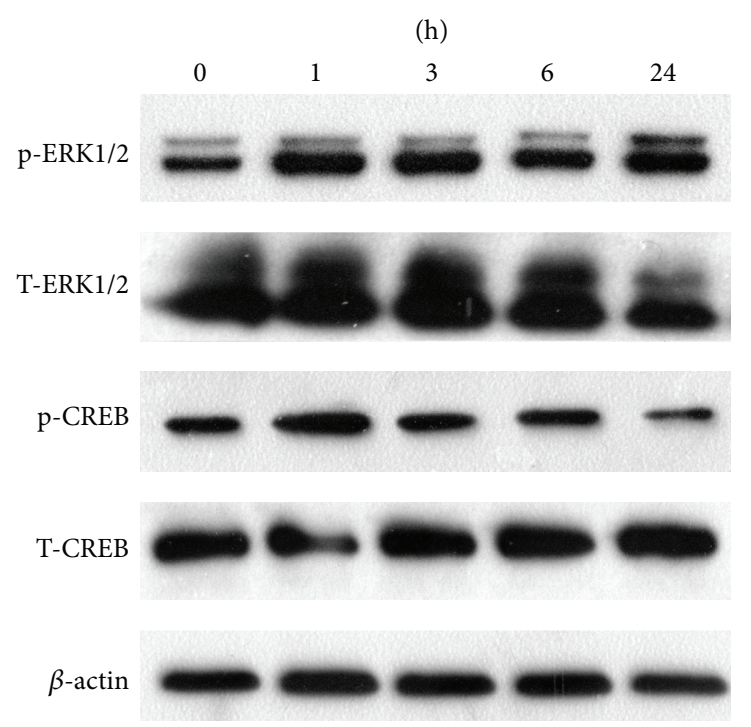

(a)

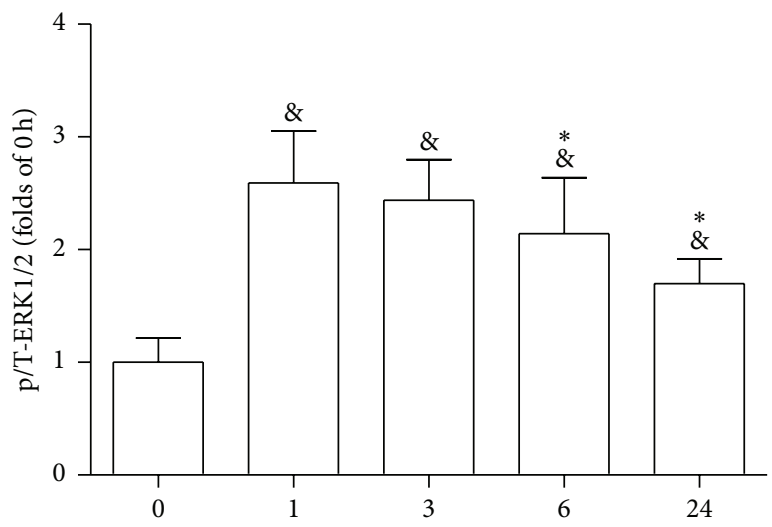

(h)

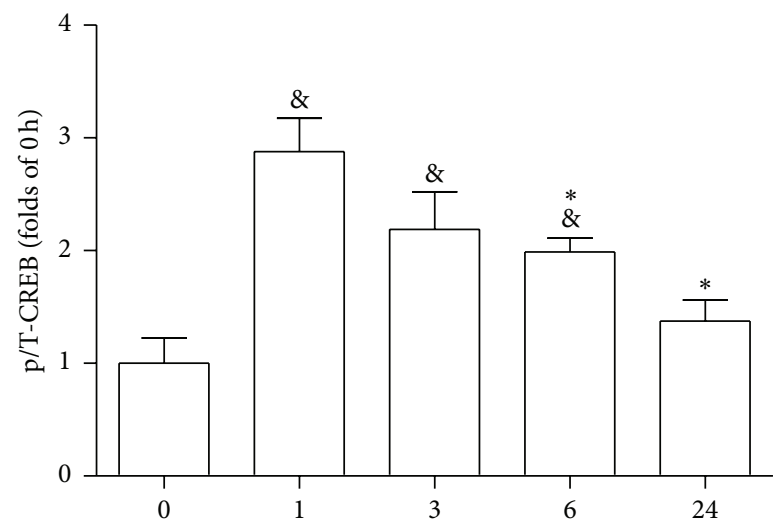

(h)

(b)

(c)

FIGURE 6: (a) Western blotting was performed to evaluate the expression levels of p-ERK1/2 and p-CREB. Brain tissues were extracted at $0 \mathrm{~h}$, $1 \mathrm{~h}, 3 \mathrm{~h}, 6 \mathrm{~h}$, and $24 \mathrm{~h}$ after MCAO. The phosphorylation of ERK1/2 (b) and CREB (c) peaked at $1 \mathrm{~h}$. Data are expressed as mean \pm SD; $n=3$ rats/group $\left({ }^{\&} P<0.05\right.$ versus $0 \mathrm{~h} ;{ }^{*} \mathrm{P}<0.05$ versus $\left.1 \mathrm{~h}\right)$.

and this phenomenon is consistent with a report demonstrating that B1R-knockout nondiabetic mice suffering brain I/R injury have smaller infarct volumes and less postischemic inflammation [34]. Therefore, a combination of the B1R antagonist with TK treatment may provide a novel strategy for the treatment of stroke with diabetes. Additionally, simultaneous antagonism of $\mathrm{B} 1 \mathrm{R}$ and $\mathrm{B} 2 \mathrm{R}$ reversed the beneficial effects of TK, suggesting that the protection induced by TK was mediated primarily by B2R, although B1R activated by TK showed a detrimental effect on brain I/R injury in diabetic rats [22]. Thus, our results demonstrated that activation of $\mathrm{B} 2 \mathrm{R}$ in cerebral $\mathrm{I} / \mathrm{R}$ damage in diabetic rats suppressed inflammation, enhanced cell survival, and improved neurological functions, whereas activation of B1R produced the opposite effects.

A previous study on myocardial ischemia demonstrated a cardioprotective effect of the $\mathrm{B} 2 \mathrm{R}$ agonist in nondiabetic mice and the B1R agonist in diabetes via inhibition of GSK-3 $\beta$ [35]. Possible explanations for this discrepancy include differences in animal species, tissues, organs, and I/R injury models. Moreover, in our findings, inactivation of B2R before MCAO resulted in a lower level of ERK1/2 phosphorylation than TK intervention alone in the ischemic brain, which was contrary to B1R inactivation. These observations suggest that activation of $\mathrm{B} 2 \mathrm{R}$ signaling induced by $\mathrm{TK}$ leads to the phosphorylation of ERK1/2 in the diabetic cerebrum, indicating that $\mathrm{B} 2 \mathrm{R}$ may be the main upstream protein in the ERK1/2 signaling pathway activated by TK.

The ERK1/2 pathway is known to be activated by various stimuli, such as oxidative stress, ionic imbalance, activation of glutamate receptors, and growth factors [36], and this pathway also plays a role in TK-mediated protection against I/R injury in orthoglycemic conditions [17-19]. Previous studies have demonstrated that the stimulation of B2R caused NO 


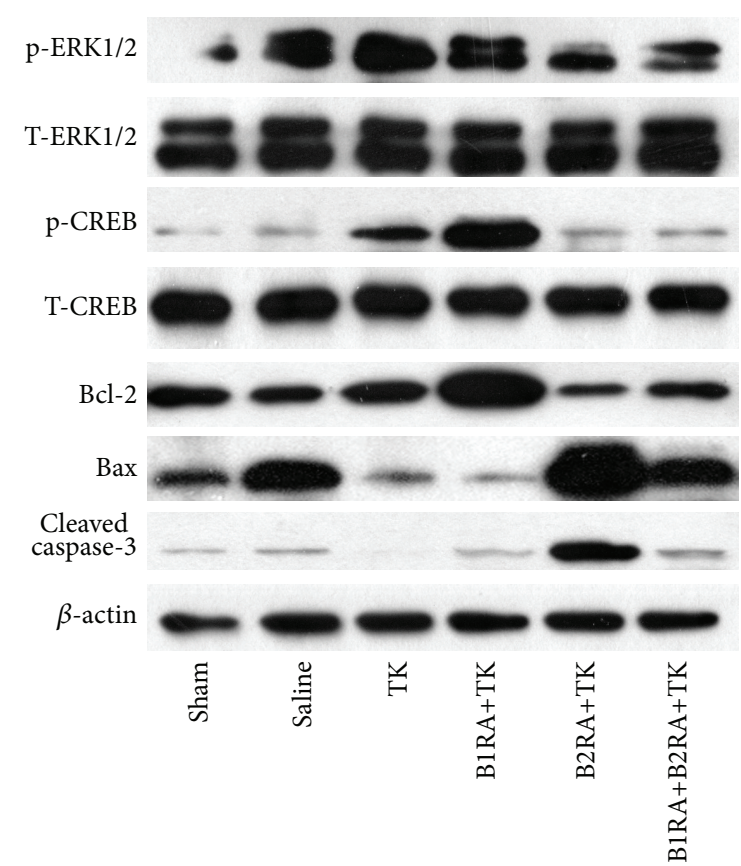

(a)

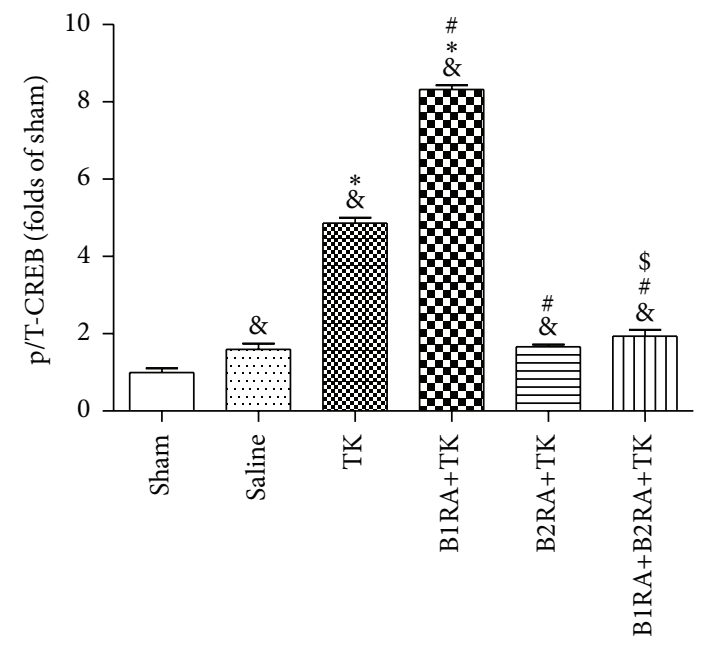

(c)

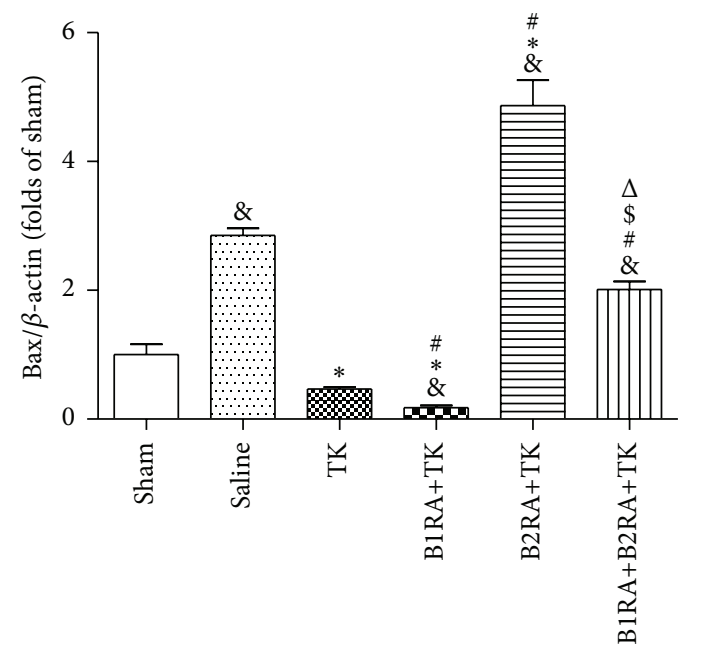

(e)

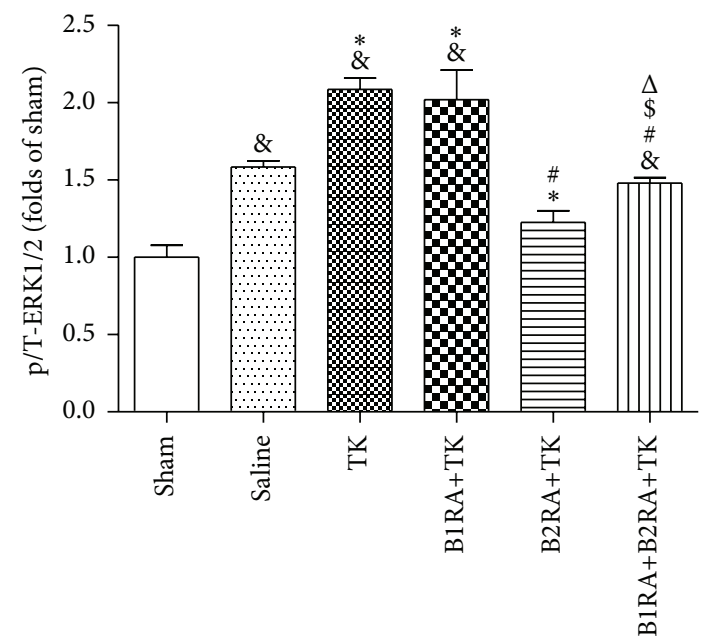

(b)

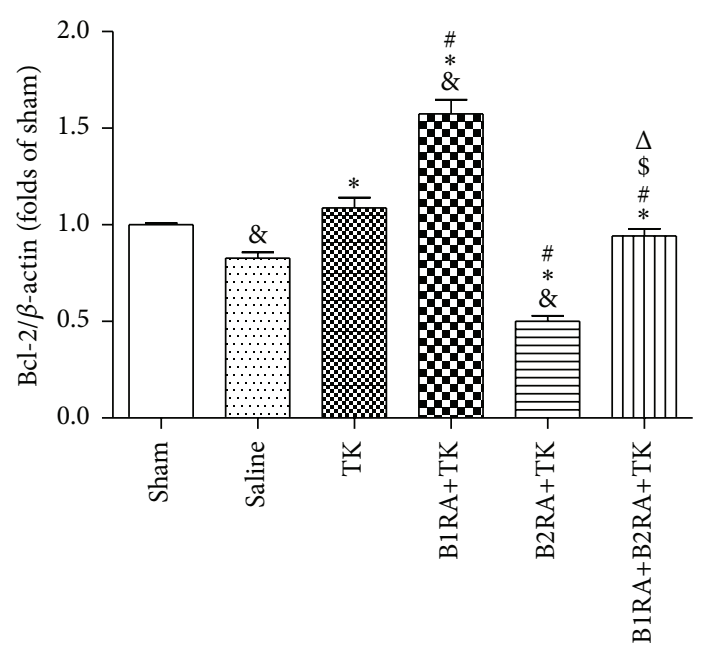

(d)

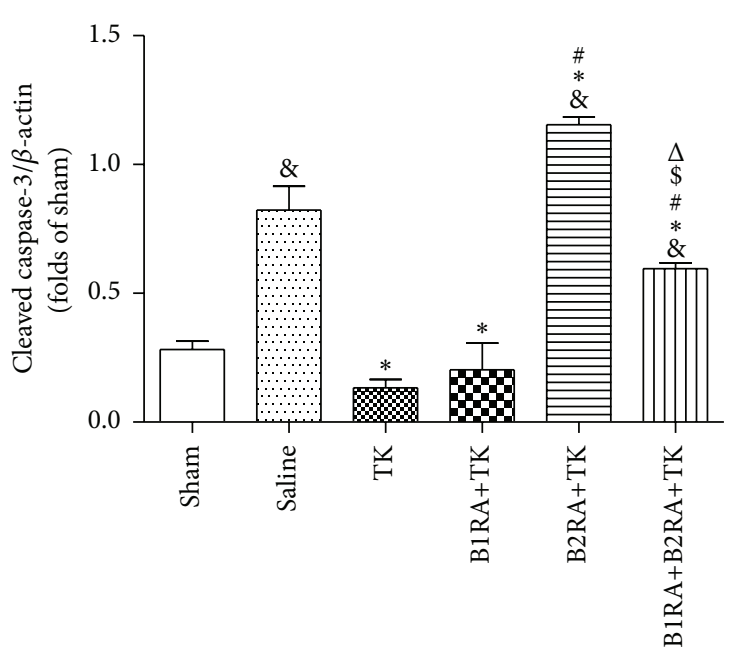

(f)

Figure 7: Continued. 


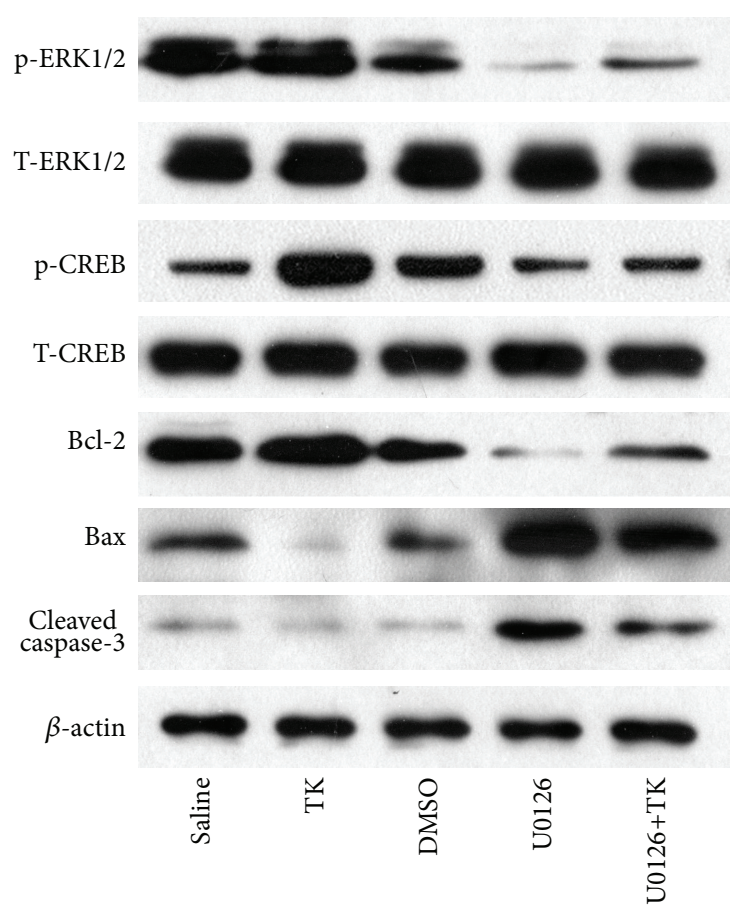

(g)

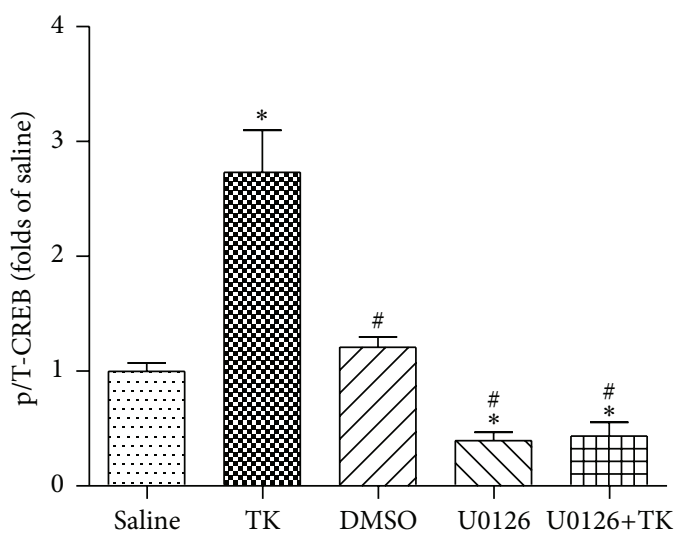

(i)

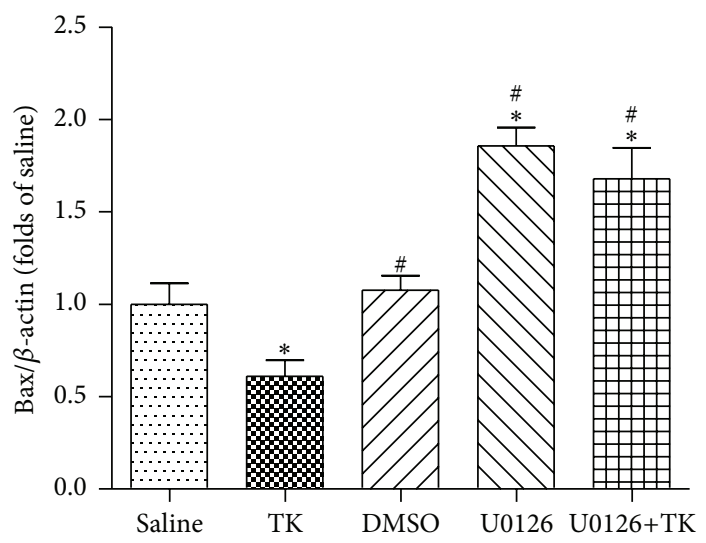

(k)

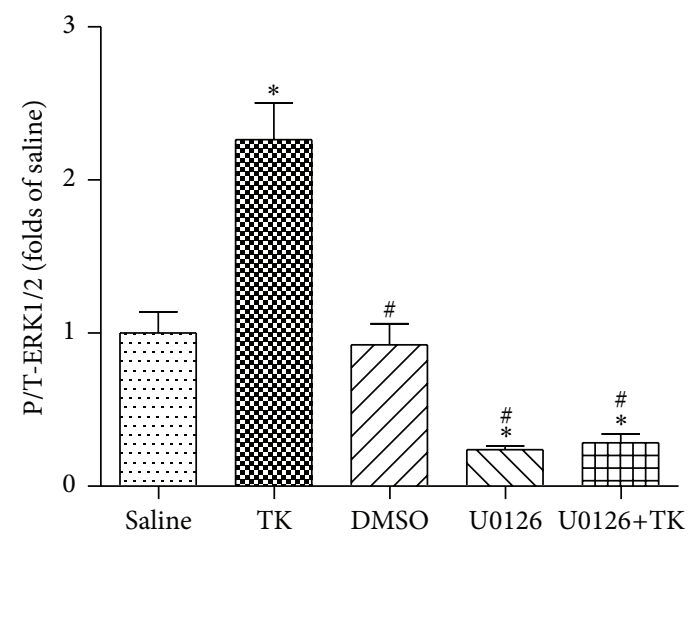

(h)

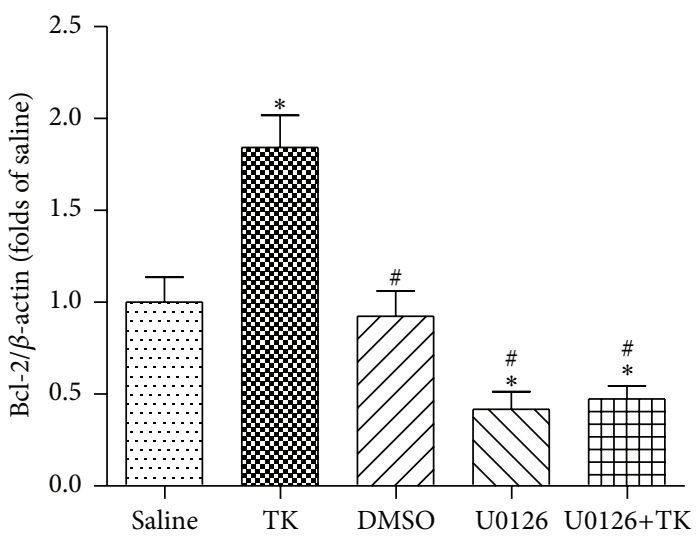

(j)

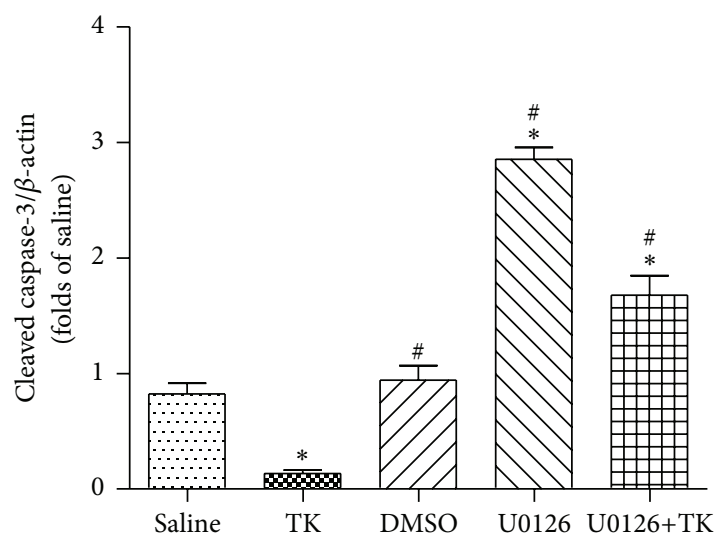

(l)

FIGURE 7: (a) Western blotting was performed to evaluate the activation levels of p-ERK1/2, p-CREB, Bcl-2, and Bax. The levels of p-ERK1/2 (b), p-CREB (c), Bcl-2 (d), Bax (e), and cleaved caspase-3 (f) are expressed as mean \pm SD; $n=4$. (g) Western blotting was performed to evaluate the activation levels of p-ERK1/2, p-CREB, Bcl-2, and Bax. The levels of p-ERK1/2 (h), p-CREB (i), Bcl-2 (j), Bax (k), and cleaved caspase-3 (l) are expressed as mean $\pm \mathrm{SD} ; n=3\left({ }^{\circledR} P<0.05\right.$ versus sham group; ${ }^{*} P<0.05$ versus saline group; ${ }^{\#} P<0.05$ versus TK group; ${ }^{\$} P<0.05$ versus B1RA+TK group; ${ }^{\Delta} P<0.05$ versus B2RA+TK group). 
production and NO could stimulate ERK $[37,38]$. Therefore, the activation of $\mathrm{B} 2 \mathrm{R}$ may lead to the upregulation of $\mathrm{p}$ ERK1/2. Here, we also observed that the ERK1/2 pathway was one of the main signaling pathways involved in TK-mediated anti-inflammation and antiapoptosis following cerebral I/R in diabetes. TK treatment during reperfusion after MCAO in diabetic rats induced upregulation of $\mathrm{p}$-ERK1/2, whereas pretreatment with the ERK1/2 pathway inhibitor U0126 before MCAO produced adverse effects that were similar to those for the B2R antagonist. U0126 was reported to significantly aggravate neurological dysfunction, increase infarct volumes and edema, and exacerbate inflammation and apoptosis [39, 40]. Our findings further showed that pretreatment with U0126 could partially but significantly counteract the efficacy of TK against cerebral I/R injury in diabetic rats. This phenomenon hints that the actions of TK against brain I/R injury in diabetic rats might involve other pathways besides the ERK1/2 signaling pathway. However, the ERK1/2 inhibitor completely suppressed the activation of $\mathrm{p}$-ERK1/2, $\mathrm{p}$-CREB, and $\mathrm{Bcl}-2$ and promoted the activation of Bax and cleaved caspase- 3 in TK-treated group, indicating that ERK1/2 may be the main upstream protein in this signaling pathway activated by TK.

$\mathrm{CREB}$, as a transcription factor, participates in synaptic plasticity, memory. and survival. Phosphorylation of CREB by $\mathrm{p}$-ERK1/2 not only displays neuroprotection in stroke animals $[36,41]$ but also prevents postischemic inflammation and neuronal damage [42]. Our data showed that the level of phosphorylated CREB changed with the expression of p-ERK1/2. Phosphorylated CREB was reported to stimulate expression of the antiapoptotic protein $\mathrm{Bcl}-2$ and the reduction of caspase-3, which are associated with neuronal survival [43-46]. Bax and Bcl-2 are members of the Bcl-2 protein family and are associated with neuronal apoptosis. Our study revealed that TK could stimulate the signal pathway of ERK1/2-CREB-Bcl-2 and suppress the Bax and cleaved caspase- 3 expressions. Additionally, pretreatment with the B1R antagonist strengthened the actions of TK on the abovementioned signaling pathways, whereas the B2R antagonist or ERK1/2 inhibitor U0126 produced the opposite effects on these signaling proteins. We also noticed sharp increases in degenerative and apoptotic neurons as well as inflammatory reactions after inhibition of the B2R or ERK1/2 pathway. The present findings were consistent with a previous study in which U0126 was shown to counteract the neuroprotection of ERK-CREB in transient global ischemia [47]. It is likely that the anti-inflammatory activity induced by TK through B2R involves enhanced phosphorylation of ERK1/2 and subsequently increases the phosphorylation of CREB, which in turn suppresses proinflammatory cytokine secretion in the damaged tissue and reduces microglia and neutrophil migration. According to our observations, TK might produce antiapoptotic effects through B2R and activation of the ERK1/2-CREB pathway, in addition to promoting Bcl-2 formation, suppressing Bax and cleaved caspase-3 expressions, and reducing cell degeneration and apoptosis.

Various drugs for stroke were neuroprotective preclinically but proved unsuccessful in clinical trials subsequently. Using healthy animals as the model to evaluate the efficacy of candidate drugs may be one of the most common problems. Recommendations issued by Stroke Therapy Academia Industry Roundtable (STAIR) Committee showed that drug intended for clinical trials should be administrated to animals accompanied with hypertension, diabetes, and hypercholesterolemia if it was demonstrated to be effective in healthy animals [46]. TK was demonstrated to offer neuroprotection by previous work in orthoglycemic models; however, the research on the role of TK in stroke animals with diabetes remains scarce. Therefore, it is meaningful to prove that TK could ameliorate the prognosis of I/R injury in hyperglycemic conditions.

In conclusion, early administration of TK could provide robust resistance to $\mathrm{I} / \mathrm{R}$ damage in the diabetic brain. This neuroprotective effect was attributed, in the present study, to the antiapoptotic and anti-inflammatory effects mediated primarily through B2R and the ERK1/2-CREB-Bcl-2 pathway. Thus, our findings support the use of TK as a therapeutic approach to reduce the effects of brain I/R insults in diabetic subjects.

\section{Competing Interests}

All authors declare that there is no conflict of interests.

\section{Authors' Contributions}

Ruifeng Shi, Kunxiong Yuan, and Bin Hu contributed equally to this work.

\section{Acknowledgments}

This research was supported by funds from the National Natural Science Foundation of China (Grants 81100870, 81471182, 81400332, and 31300900) and Natural Science Foundation of Jiangsu Province BK20141373.

\section{References}

[1] C. J. L. Murray, T. Vos, R. Lozano et al., "Disability-adjusted life years (DALYs) for 291 diseases and injuries in 21 regions, 19902010: a systematic analysis for the Global Burden of Disease study 2010," The Lancet, vol. 380, no. 9859, pp. 2197-2223, 2010.

[2] M. J. O’Donnell, D. Xavier, L. S. Liu et al., "Risk factors for ischaemic and intracerebral haemorrhagic stroke in 22 countries (the INTERSTROKE study): a case-control study," The Lancet, vol. 376, no. 9735, pp. 112-123, 2010.

[3] M. J. A. Luitse, G. J. Biessels, G. E. H. M. Rutten, and L. J. Kappelle, "Diabetes, hyperglycaemia, and acute ischaemic stroke," The Lancet Neurology, vol. 11, no. 3, pp. 261-271, 2012.

[4] J. Putaala, R. Liebkind, D. Gordin et al., "Diabetes mellitus and ischemic stroke in the young: clinical features and long-term prognosis," Neurology, vol. 76, no. 21, pp. 1831-1837, 2011.

[5] R. Prakash, W. G. Li, Z. Qu, M. A. Johnson, S. C. Fagan, and A. Ergul, "Vascularization pattern after ischemic stroke is different in control versus diabetic rats relevance to stroke recovery," Stroke, vol. 44, no. 10, pp. 2875-2882, 2013. 
[6] B. M. Kissela, J. Khoury, D. Kleindorfer et al., "Epidemiology of ischemic stroke in patients with diabetes-the greater Cincinnati/Northern Kentucky stroke study," Diabetes Care, vol. 28, no. 2, pp. 355-359, 2005.

[7] T. A. Baird, M. W. Parsons, T. Phan et al., "Persistent poststroke hyperglycemia is independently associated with infarct expansion and worse clinical outcome," Stroke, vol. 34, no. 9, pp. 22082214, 2003.

[8] W. Hacke, "Interventional thrombectomy for major strokea step in the right direction," The New England Journal of Medicine, vol. 372, no. 1, pp. 76-77, 2015.

[9] J. Panés, I. Kurose, M. D. Rodriguez-Vaca et al., "Diabetes exacerbates inflammatory responses to ischemia-reperfusion," Circulation, vol. 93, no. 1, pp. 161-167, 1996.

[10] Y. Yatomi, R. Tanaka, Y. Shimada et al., “Type 2 diabetes reduces the proliferation and survival of oligodendrocyte progenitor cells in ishchemic white matter lesions," Neuroscience, vol. 289, pp. 214-223, 2015.

[11] Y. Akamatsu, Y. Nishijima, C. C. Lee et al., "Impaired leptomeningeal collateral flow contributes to the poor outcome following experimental stroke in the type 2 diabetic mice," The Journal of Neuroscience, vol. 35, no. 9, pp. 3851-3864, 2015.

[12] A. E. El-Sahar, M. M. Safar, H. F. Zaki, A. S. Attia, and A. A. Ain-Shoka, "Sitagliptin attenuates transient cerebral ischemia/reperfusion injury in diabetic rats: implication of the oxidative-inflammatory-apoptotic pathway," Life Sciences, vol. 126, pp. 81-86, 2015.

[13] D. Li, B. Huang, J. Liu, L. Li, and X. Li, "Decreased brain KATP channel contributes to exacerbating ischemic brain injury and the failure of neuroprotection by sevoflurane post-conditioning in diabetic rats," PLoS ONE, vol. 8, no. 8, Article ID e73334, 2013.

[14] C. Albert-Weißenberger, A.-L. Sirén, and C. Kleinschnitz, "Ischemic stroke and traumatic brain injury: the role of the kallikrein-kinin system," Progress in Neurobiology, vol. 101-102, pp. 65-82, 2013.

[15] L. Liu, R. L. Zhang, K. Liu et al., "Tissue kallikrein alleviates glutamate-induced neurotoxicity by activating ERK1," Journal of Neuroscience Research, vol. 87, no. 16, pp. 3576-3590, 2009.

[16] L. Liu, R. Zhang, K. Liu et al., “Tissue kallikrein protects cortical neurons against in vitro ischemia-acidosis/reperfusioninduced injury through the ERK1/2 pathway," Experimental Neurology, vol. 219, no. 2, pp. 453-465, 2009.

[17] L. Liu, H. B. Liu, F. Yang et al., "Tissue kallikrein protects cortical neurons against hypoxia/reoxygenation injury via the ERK1/2 pathway,' Biochemical and Biophysical Research Communications, vol. 407, no. 2, pp. 283-287, 2011.

[18] Z.-B. Chen, D.-Q. Huang, F.-N. Niu, X. Zhang, E.-G. Li, and $\mathrm{Y} . \mathrm{Xu}$, "Human urinary kallidinogenase suppresses cerebral inflammation in experimental stroke and downregulates nuclear factor- $\kappa$ B," Journal of Cerebral Blood Flow \& Metabolism, vol. 30, no. 7, pp. 1356-1365, 2010.

[19] Z. Wang, X. Han, M. Cui, K. Fang, Z. Y. Lu, and Q. Dong, "Tissue kallikrein protects rat hippocampal CA1 neurons against cerebral ischemia/reperfusion-induced injury through the B2R-Raf-MEK1/2-ERK1/2 pathway," Journal of Neuroscience Research, vol. 92, no. 5, pp. 651-657, 2014.

[20] G. Yuan, J. Deng, T. Wang et al., “Tissue kallikrein reverses insulin resistance and attenuates nephropathy in diabetic rats by activation of phosphatidylinositol 3-kinase/protein kinase $\mathrm{B}$ and adenosine 5'-monophosphate-activated protein kinase signaling pathways," Endocrinology, vol. 148, no. 5, pp. 20162026, 2007.
[21] D. J. Campbell, A. Kladis, Y. Zhang et al., "Increased tissue kallikrein levels in type 2 diabetes," Diabetologia, vol. 53, no. 4 , pp. 779-785, 2010.

[22] H. Sang, L. Liu, L. Wang et al., "Opposite roles of bradykinin B1 and $\mathrm{B} 2$ receptors during cerebral ischaemia-reperfusion injury in experimental diabetic rats," European Journal of Neuroscience, vol. 43, no. 1, pp. 53-65, 2016.

[23] K. Srinivasan, B. Viswanad, L. Asrat, C. L. Kaul, and P. Ramarao, "Combination of high-fat diet-fed and low-dose streptozotocintreated rat: a model for type 2 diabetes and pharmacological screening," Pharmacological Research, vol. 52, no. 4, pp. 313-320, 2005.

[24] E. Z. Longa, P. R. Weinstein, S. Carlson, and R. Cummins, "Reversible middle cerebral artery occlusion without craniectomy in rats," Stroke, vol. 20, no. 1, pp. 84-91, 1989.

[25] L. Ling, Q. Hou, S. Xing, J. Yu, Z. Pei, and J. Zeng, "Exogenous kallikrein enhances neurogenesis and angiogenesis in the subventricular zone and the peri-infarction region and improves neurological function after focal cortical infarction in hypertensive rats," Brain Research, vol. 1206, pp. 89-97, 2008.

[26] Y. X. Lu, J. S. Kang, Y. Bai et al., "Hyperbaric oxygen enlarges the area of brain damage in MCAO rats by blocking autophagy via ERK1/2 activation," European Journal of Pharmacology, vol. 728, no. 1, pp. 93-99, 2014.

[27] J. Chen, Y. Li, L. Wang et al., "Therapeutic benefit of intravenous administration of bone marrow stromal cells after cerebral ischemia in rats," Stroke, vol. 32, no. 4, pp. 1005-1011, 2001.

[28] K. J. Livak and T. D. Schmittgen, "Analysis of relative gene expression data using real-time quantitative PCR and the 2$\Delta \Delta$ CT method," Methods, vol. 25, no. 4, pp. 402-408, 2001.

[29] F. Guo, T. Jiang, W. Song et al., "Electroacupuncture attenuates cerebral ischemia-reperfusion injury in diabetic mice through adiponectin receptor 1-mediated phosphorylation of GSK-3 $\beta$," Molecular Neurobiology, vol. 51, no. 2, pp. 685-695, 2015.

[30] J. Huang, B. Liu, C. Yang, H. Chen, D. Eunice, and Z. Yuan, "Acute hyperglycemia worsens ischemic stroke-induced brain damage via high mobility group box-1 in rats," Brain Research, vol. 1535, pp. 148-155, 2013.

[31] S. Suda, M. Ueda, C. Nito et al., "Valproic acid ameliorates ischemic brain injury in hyperglycemic rats with permanent middle cerebral occlusion," Brain Research, vol. 1606, pp. 1-8, 2015.

[32] C.-F. Xia, R. S. Smith Jr., B. Shen et al., "Postischemic brain injury is exacerbated in mice lacking the kinin B2 receptor," Hypertension, vol. 47, no. 4, pp. 752-761, 2006.

[33] C.-F. Xia, H. Yin, Y.-Y. Yao, C. V. Borlongan, L. Chao, and J. Chao, "Kallikrein protects against ischemic stroke by inhibiting apoptosis and inflammation and promoting angiogenesis and neurogenesis," Human Gene Therapy, vol. 17, no. 2, pp. 206-219, 2006.

[34] M. Austinat, S. Braeuninger, J. B. Pesquero et al., "Blockade of bradykinin receptor b1 but not bradykinin receptor B2 provides protection from cerebral infarction and brain edema," Stroke, vol. 40, no. 1, pp. 285-293, 2009.

[35] L. Potier, L. Waeckel, M.-P. Vincent et al., "Selective kinin receptor agonists as cardioprotective agents in myocardial ischemia and diabetes," Journal of Pharmacology and Experimental Therapeutics, vol. 346, no. 1, pp. 23-30, 2013.

[36] S. L. Mehta, N. Manhas, and R. Raghubir, "Molecular targets in cerebral ischemia for developing novel therapeutics," Brain Research Reviews, vol. 54, no. 1, pp. 34-66, 2007. 
[37] H. M. Lander, A. T. Jacovina, R. J. Davis, and J. M. Tauras, "Differential activation of mitogen-activated protein kinases by nitric oxide-related species," Journal of Biological Chemistry, vol. 271, no. 33, pp. 19705-19709, 1996.

[38] J. Chao, G. Bledsoe, H. Yin, and L. Chao, "The tissue kallikreinkinin system protects against cardiovascular and renal diseases and ischemic stroke independently of blood pressure reduction," Biological Chemistry, vol. 387, no. 6, pp. 665-675, 2006.

[39] X.-K. Tu, W.-Z. Yang, J.-P. Chen et al., "Repetitive ischemic preconditioning attenuates inflammatory reaction and brain damage after focal cerebral ischemia in rats: involvement of PI3K/Akt and ERK1/2 signaling pathway," Journal of Molecular Neuroscience, vol. 55, no. 4, pp. 912-922, 2015.

[40] X. Xu, L. Tu, W. Jiang, W. Feng, C. X. Zhao, and D. W. Wang, "Bradykinin prevents the apoptosis of NIT-1 cells induced by TNF- $\alpha$ via the PI3K/Akt and MAPK signaling pathways," International Journal of Molecular Medicine, vol. 29, no. 5, pp. 891-898, 2012.

[41] H.-T. Lee, Y.-C. Chang, L.-Y. Wang, S.-T. Wang, C.-C. Huang, and C.-J. Ho, "cAMP response element-binding protein activation in ligation preconditioning in neonatal brain," Annals of Neurology, vol. 56, no. 5, pp. 611-623, 2004.

[42] J.-H. Yi, S.-W. Park, R. Kapadia, and R. Vemuganti, "Role of transcription factors in mediating post-ischemic cerebral inflammation and brain damage," Neurochemistry International, vol. 50, no. 7-8, pp. 1014-1027, 2007.

[43] F. Wu, R. Echeverry, J. Wu et al., "Tissue-type plasminogen activator protects neurons from excitotoxin-induced cell death via activation of the ERK1/2-CREB-ATF3 signaling pathway," Molecular and Cellular Neuroscience, vol. 52, pp. 9-19, 2013.

[44] W.-Y. Lin, Y.-C. Chang, H.-T. Lee, and C.-C. Huang, "CREB activation in the rapid, intermediate, and delayed ischemic preconditioning against hypoxic-ischemia in neonatal rat," Journal of Neurochemistry, vol. 108, no. 4, pp. 847-859, 2009.

[45] L. Wang, S. Deng, Y. Lu et al., "Increased inflammation and brain injury after transient focal cerebral ischemia in activating transcription factor 3 knockout mice," Neuroscience, vol. 220, pp. 100-108, 2012.

[46] W. Huang, X. Liu, J. Cao et al., "miR-134 regulates ischemia/ reperfusion injury-induced neuronal cell death by regulating CREB signaling," Journal of Molecular Neuroscience, vol. 55, no. 4, pp. 821-829, 2015.

[47] E. M. Park, T. H. Joh, B. T. Volpe, C. K. Chu, G. Song, and S. Cho, "A neuroprotective role of extracellular signal-regulated kinase in $\mathrm{N}$-acetyl-O-methyldopamine-treated hippocampal neurons after exposure to in vitro and in vivo ischemia," Neuroscience, vol. 123, no. 1, pp. 147-154, 2004. 


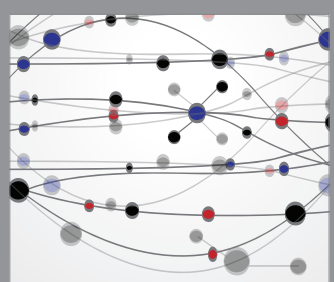

The Scientific World Journal
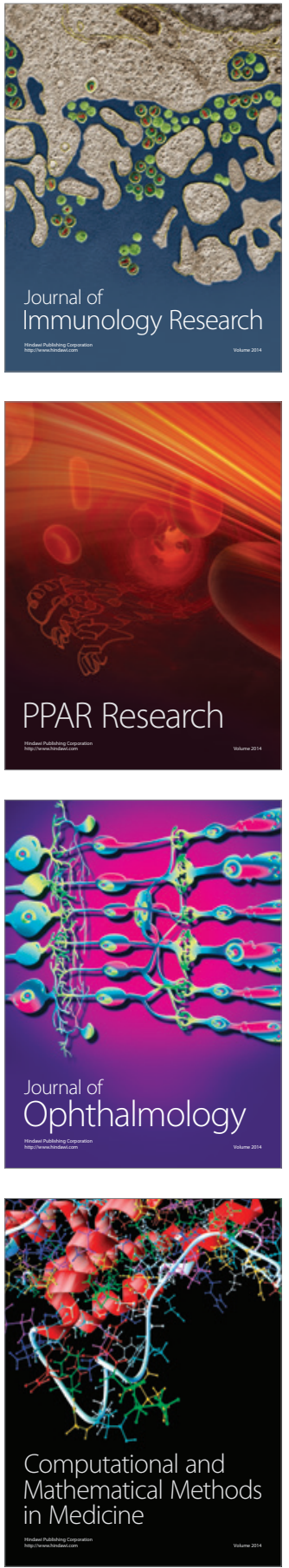

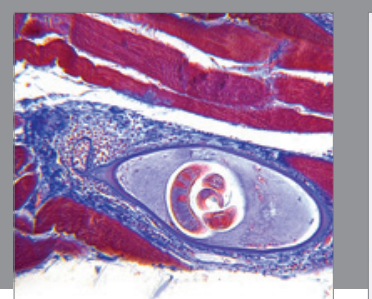

Gastroenterology Research and Practice

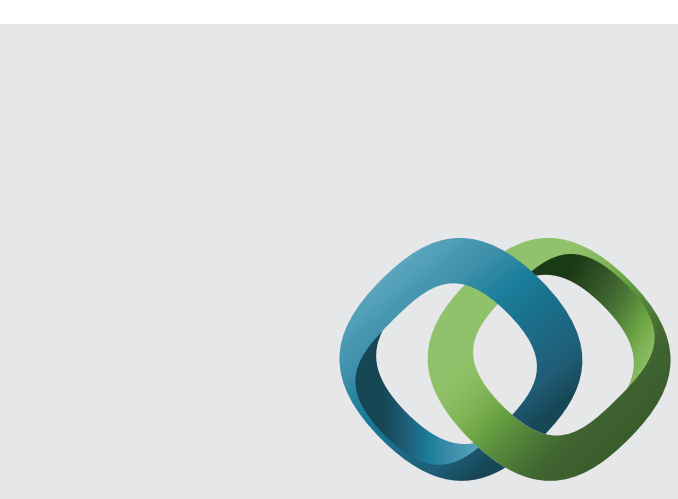

\section{Hindawi}

Submit your manuscripts at

http://www.hindawi.com
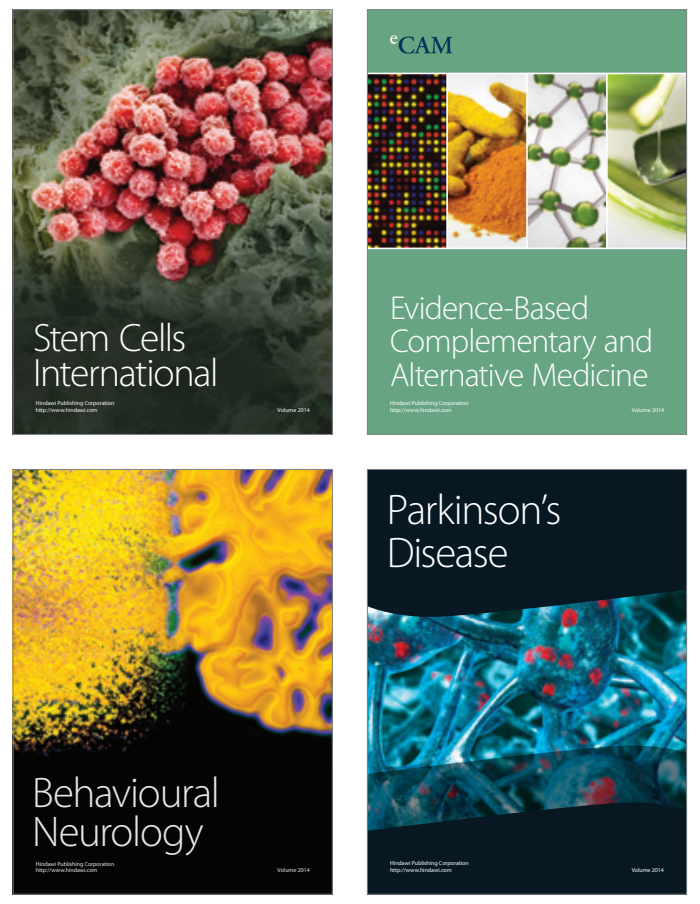
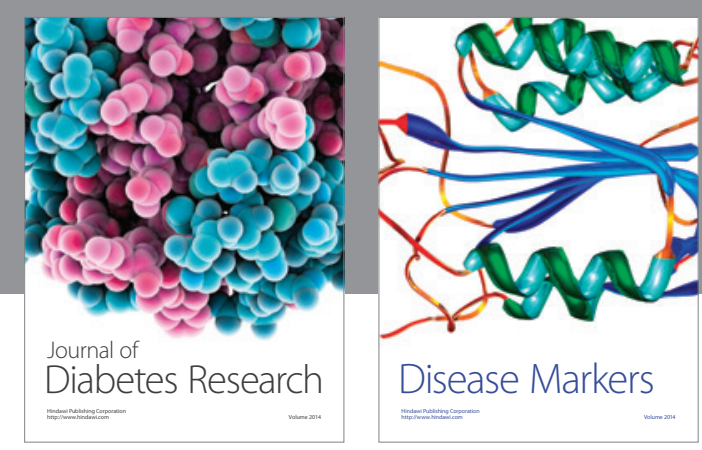

Disease Markers
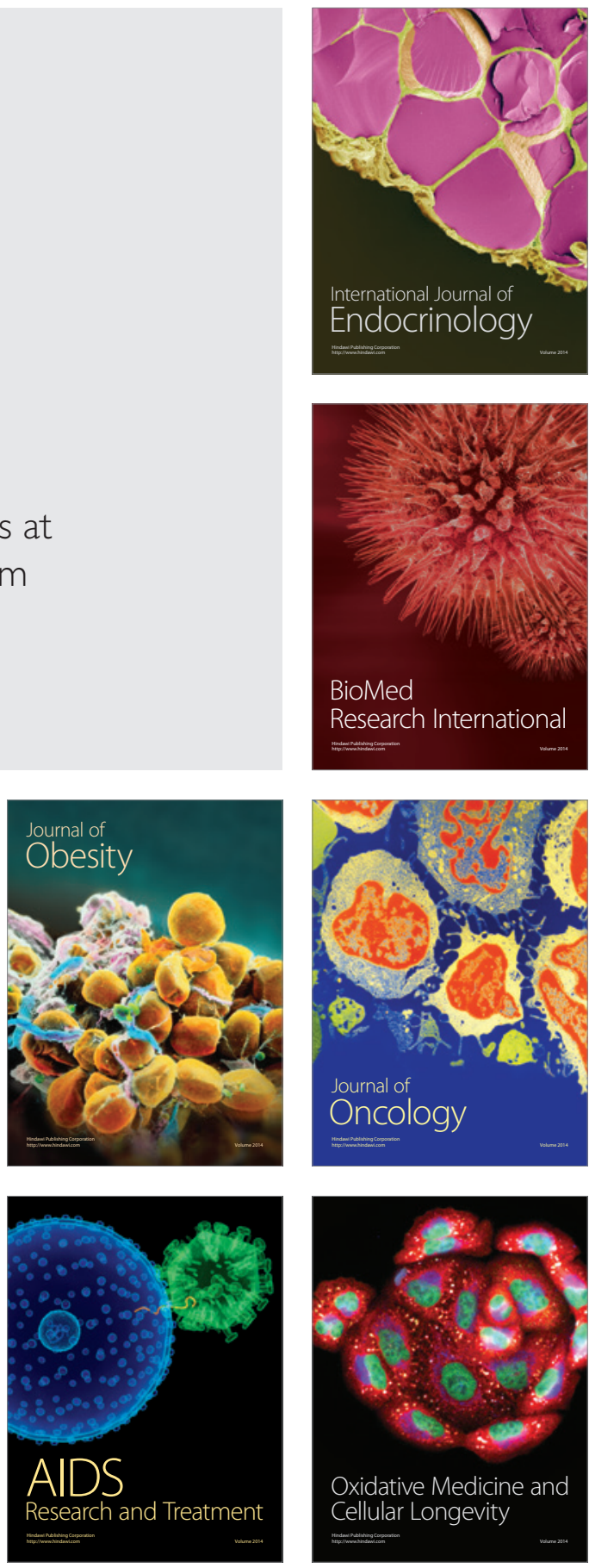